\title{
An Overview of Regulation Topologies in Resonant Wireless Power Transfer Systems for Consumer Electronics or Bio-Implants
}

\author{
Yang Liu ${ }^{\mathbb{D}}$, Bin Li, Mo Huang *, Zhijian Chen and Xiuyin Zhang \\ School of Electronics and Information Engineering, South China University of Technology, \\ Wushan, Guangzhou 510641, China; eeyangliu1993@mail.scut.edu.cn (Y.L.); phlibin@scut.edu.cn (B.L.); \\ chenzhijian@scut.edu.cn (Z.C.); zhangxiuyin@scut.edu.cn (X.Z.) \\ * Correspondence: mohuang@scut.edu.cn; Tel.: +86-020-2223-6016
}

Received: 31 May 2018; Accepted: 25 June 2018; Published: 3 July 2018

\begin{abstract}
Owing to its relatively high efficiency, extended transmission range, and less exposure to radio frequency radiation, near-field resonant wireless power transfer (R-WPT) has been widely used in consumer electronics and bio-implants. For most applications, a well-regulated output voltage is required against the coupling and loading variations, and thus a regulation scheme should be employed in an R-WPT system. To achieve an optimal receiver (RX) or overall efficiency, together with a reduced cost overhead, several regulation schemes have been proposed in recent years, where the regulation can be implemented at either the RX or transmitter (TX) side, or both. These regulation schemes have been reviewed and comprehensively discussed in this paper. Hence, the main contribution of this paper is to provide a guideline for designing the regulation scheme in R-WPT systems. Moreover, potential new topologies of regulation are investigated here.
\end{abstract}

Keywords: resonant wireless power transfer (R-WPT); regulation; rectifier; power amplifier (PA); receiver; transmitter

\section{Introduction}

The wireless power transfer (WPT) technique is at the critical point of explosive growth. Many consumer electronics and bio-implant systems have integrated WPT circuits for their compactness, being water-proof, and their reduced maintenance cost. However, the WPT circuits' transfer efficiency is inherently lower than that of their wired counterparts [1,2]. To balance this trade-off, high-efficiency WPT techniques, e.g., near-field transmission (such as inductive, resonant, capacitive, and ultrasonic power transfer), may be more favorable than far-field WPT (such as optical and microwave). In addition, the near-field technique advances far-field WPT in terms of less exposure to radio frequency (RF) radiation. Among the near-field WPT techniques, resonant WPT (R-WPT) has gradually drawn widespread attention. This is because the R-WPT technique can extend the power transfer range to several tens of centimeters, which is much wider than that of the inductive coupling technique. On one other hand, the resonant coupling technique does not require a precise coil alignment and tight coupling, and thus multiple receivers can be applied to receive power from a transmitter.

Some previous studies have reviewed the R-WPT techniques [3-7]. In [3,4], the historical background, challenges, and engineering applications of R-WPT were discussed. The authors in [5] presented the coil design challenges. A comparison between the inductive and capacitive power transfer in power level, gap distance, operating frequency, and efficiency were given in [6]. In addition, [7] discussed the design of resonant circuits and the compensation networks for near-field WPT systems. 
Taking an R-WPT system with series-resonant inductor and capacitor (LC) tanks as an example, as illustrated in Figure 1, it consists of a transmitter (TX), a wireless link, and a receiver (RX). In general, the TX is composed of a direct current (DC)-DC converter for power supply, gate drivers, and a power amplifier (PA), where the VDD is the supply voltage; the wireless link consists of two series $L C$ tanks ( $L_{\mathrm{TX}} C_{\mathrm{TX}}$ and $L_{\mathrm{RX}} C_{\mathrm{RX}}$ ) resonant in the same angular frequency $\omega_{\text {res, }}$, where $k$ is the coupling coefficient between $L_{T X}$ and $L_{R X}$; and the RX is composed of a rectifier. The alternating current (AC) power, generated from the PA, is transferred from the $L_{T X}$ to the $L_{R X}$, where the AC voltage across the $L_{R X}$ is $V_{\mathrm{R}}$ and the equivalent resistance seen from the RX is $R_{\mathrm{eq}}$. Then, the rectifier converts the $V_{\mathrm{R}}$ into a DC voltage $V_{\text {OUT }}$ for a given load $R_{\mathrm{L}}$.

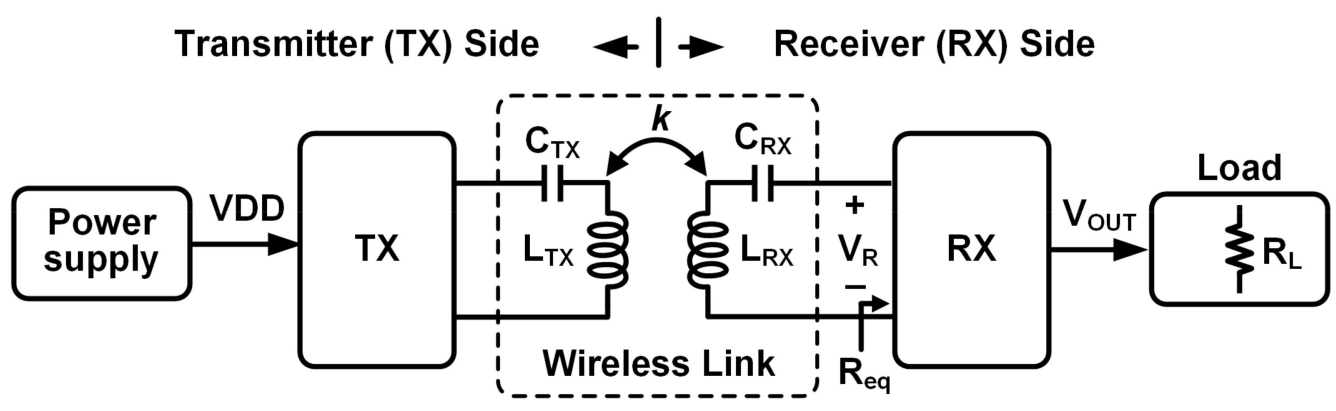

Figure 1. The block diagram of a resonant wireless power transfer (R-WPT) system with series-resonant transmitter (TX) and receiver (RX) tanks.

There are four important parameters for this WPT system: (1) the power delivered to the load (PDL) equaling to $V_{\text {OUT }}^{2} / R_{\mathrm{L}}$; (2) the power transmission efficiency (PTE) PDL $/ P_{\mathrm{S}}$, where $P_{\mathrm{S}}$ is the TX input power; (3) the power conversion efficiency (PCE) PDL $/ P_{\mathrm{R}}$, where $P_{\mathrm{R}}$ is the RX input power; and (4) the voltage conversion efficiency (VCE) $V_{\mathrm{OUT}} / V_{\mathrm{R}}$.

For most of the applications, the power supply should be well-regulated. For instance, the power supply should be regulated to $>10 \mathrm{~V}$ for the neural stimulators in implantable medical devices (IMDs) [8] or flash memory circuits [9]. For battery charging, a constant current and voltage control is required [10]. Also, the regulation becomes especially important in an R-WPT system considering load and coupling distance variation and a coil misalignment [11]. Additionally, a poorly designed regulation scheme may degrade the PTE. Therefore, a comprehensive review of regulation in R-WPT is essential.

How can the regulation be implemented in an R-WPT system? It is straightforward to fulfill the regulation at the RX side, but it can also be fulfilled at the TX side. For clarification, a simplified circuit model of an R-WPT system is presented in Figure 2a without considering the coil resistance. The PA output can be modeled as a source $V_{\mathrm{PA}}$ with a source resistor $R_{\mathrm{S}}$. As shown in Figure $2 \mathrm{~b}$, the effect of the RX's resonant magnetic coupling with the TX is presented as a reflected resistance $R_{\text {refl }}$ in series with the $L_{\mathrm{TX}}$, which can be written by

$$
R_{\text {refl }}=k^{2} \omega_{\text {res }} L_{\mathrm{TX}} Q_{\text {load }}
$$

where $Q_{\text {load }}$ is the loaded Q-factor of the $\mathrm{RX}$ resonant tank, which is given by:

$$
Q_{\text {load }}=\left\{\begin{array}{l}
\frac{\omega L_{\mathrm{RX}}}{R_{\mathrm{eq}}} \text { series }- \text { resonantRXtank } \\
\omega C_{\mathrm{RX}} R_{\text {eq }} \text { parallel }- \text { resonantRXtank. }
\end{array} .\right.
$$

It should be noted that the power received by the $\mathrm{RX}$ is equivalent to that which dissipates on $R_{\text {refl, }}$ which can be expressed as

$$
P_{\mathrm{R}}=V_{\text {refl }} \times I_{\mathrm{TX}}
$$


where $V_{\text {refl }}$ is the voltage across $R_{\text {refl. }}$. Based on Equation (3), once $V_{\text {refl }}$ or $I_{\mathrm{TX}}$ is regulated at the TX side, the received power can be regulated as well as $V_{\text {OUT }}$. Therefore, regulation can be achieved at the TX side.
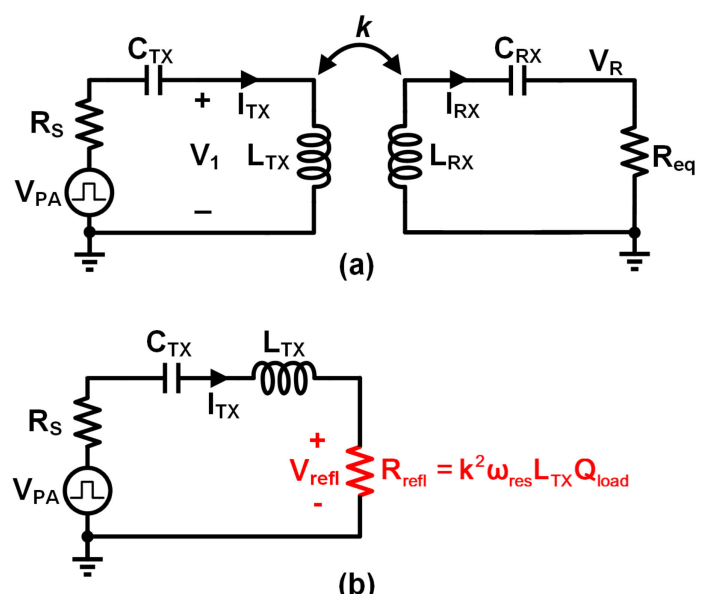

(b)

Figure 2. (a) The circuit model of the R-WPT system with series-resonant TX and RX tanks, and (b) the effect of the RX's resonant magnetic coupling with the TX is presented as a reflected resistance in the TX.

Therefore, the regulation schemes investigated in the previous works can also be categorized into two types: RX and TX regulation. As illustrated in Figure 3, RX regulation includes post-stage regulation (achieved by a cascading low dropout regulator (LDO [12-15] or a DC-DC converter [16-20]) and one-stage regulation, including active diode conduction time control [21-23], reconfigurable regulating rectification [24-27], and switching-based current-mode regulation [28-31]. On the other hand, TX regulation can be achieved by PA supply voltage control [32,33], resonant frequency control [34], PA power switch duty cycle control [35-40], and vector power summing control [41,42]. Additionally, switching frequency modulation [35,36], pulse density modulation [37,38], and phase-shifted modulation $[39,40]$ can be classified as power switch duty cycle control.
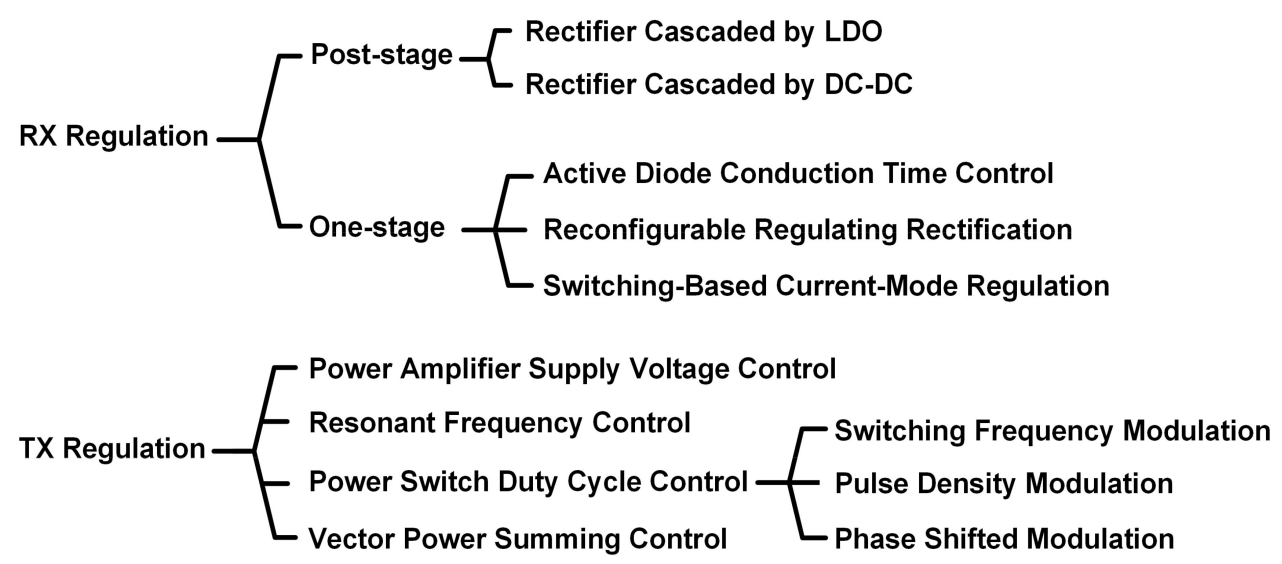

Figure 3. The category of the regulation topologies. LDO, low dropout regulator; DC, direct current.

This paper is organized as follows: Section 2 reviews the operation principles of the previous $\mathrm{RX}$ regulation topologies. Then, the operation principles of $\mathrm{TX}$ regulation are reviewed in Section 3. Possible combinations and potential new topologies are given in Section 4. Finally, a conclusion is drawn in Section 5. 


\section{RX Regulation}

\subsection{Post-Stage Regulation}

At the RX side, post-stage regulation is straightforward and has been widely used [12-20]. This scheme adopts a rectifier cascaded by a regulating stage as shown in Figure 4 . Both the LDO [12-15] and the DC-DC converter [16-20] can be implemented as the cascaded stage. These schemes are discussed as follows.

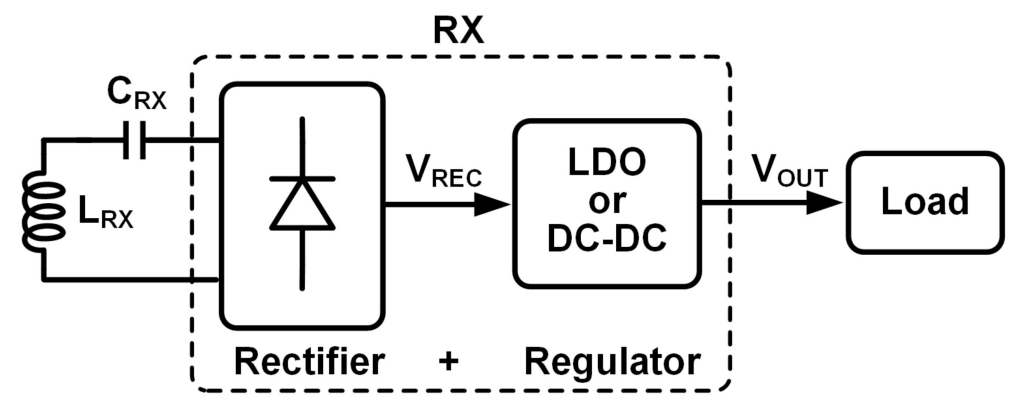

Figure 4. The block diagram of an RX with post-stage regulation.

\subsubsection{The Rectifier Cascaded by an LDO}

An LDO cascading the rectifier can provide precise regulation while keeping a small voltage ripple compared to a DC-DC converter. For example, as in Figure 5, three LDOs are cascading the rectifier in [12], supplying the analog, digital, and reference circuits, respectively, in a system-on-a-chip (SoC). Additionally, due to the high isolation among LDOs, each regulation loop can be independently optimized. However, the PCE is inherently low due to the LDOs' efficiency being directly determined by the ratio of the output and input voltages. Therefore, the maximum rectifier efficiency and PCE of [12] was $85 \%$ and $74.8 \%$, respectively, which is limited by the efficiency of the post-regulation stage.

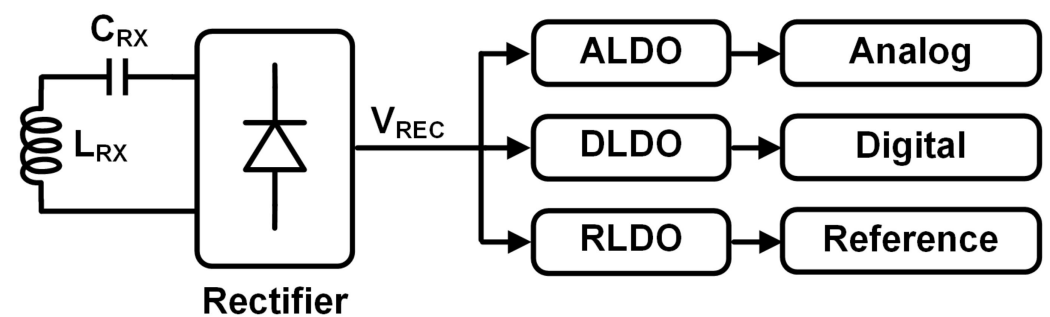

Figure 5. The RX with cascaded LDOs [12]. ALDO, analog low dropout regulator; DLDO, digital low dropout regulator; RLDO, reference low dropout regulator.

\subsubsection{The Rectifier Cascaded by a DC-DC Converter}

The PCE can be improved by using a high-efficiency post-regulator, e.g., a DC-DC converter. The authors in [16] showed a rectifier cascaded by a buck converter for battery charging as illustrated in Figure 6. Additionally, average current-mode control (ACMC) is applied for precise load current control in both continuous and discontinuous inductor current modes. With this topology, the measured rectifier efficiency and PCE were enhanced to $92.8 \%$ and $84.6 \%$, respectively. However, additional bulky passive components, e.g., an off-chip inductor, are required in the DC-DC converter, resulting in a significant cost overhead and low integration. Moreover, the switching operation of the DC-DC converter increases the output ripple and ground noise. 


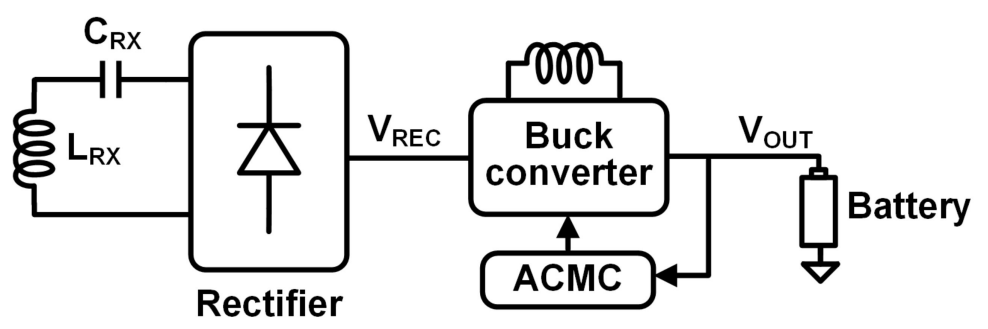

Figure 6. The RX with cascaded a buck converter [16]. ACMC, average current-mode control.

\subsubsection{The Rectifier Cascaded by an Inductorless Switching Converter}

To eliminate the bulky inductor in [16], a resonant regulating rectifier (3R) was proposed in [17]. The working principle of this 3R scheme is illustrated in Figure $7 \mathrm{a}$, where a switch $S_{1}$ is inserted between the rectifier and the load. When $S_{1}$ is turned on, the rectifier output current $I_{\text {REC }}$ charges the load (On duty in Figure 7b), while in the Off duty, $S_{1}$ is turned off and the resonant current $I_{R X}$ freewheels without charging. Then, $V_{\text {OUT }}$ can be regulated by controlling the turn-on duty cycle of $S_{1}$ using the pulse width modulation (PWM). During the off duty, the $I_{\mathrm{REC}}$ reaches zero when $S_{1}$ is turned off due to a very large equivalent resistance $R_{\text {eq. }}$. Hence, the rectifier is working in Discontinuous Conduction Mode (DCM) similar to a DC-DC converter. This feature is not desirable, since the root meam square (RMS) resonant current $I_{R X-R M S}$ in this case will be much higher than that in Continuous Conduction Mode (CCM). This results in a higher coil power loss represented by $I_{R X-R M S}{ }^{2} \times R_{2}$, where $R_{2}$ represents the $L_{R X}$ resistance.

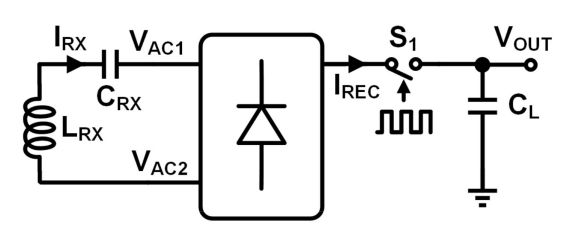

(a)

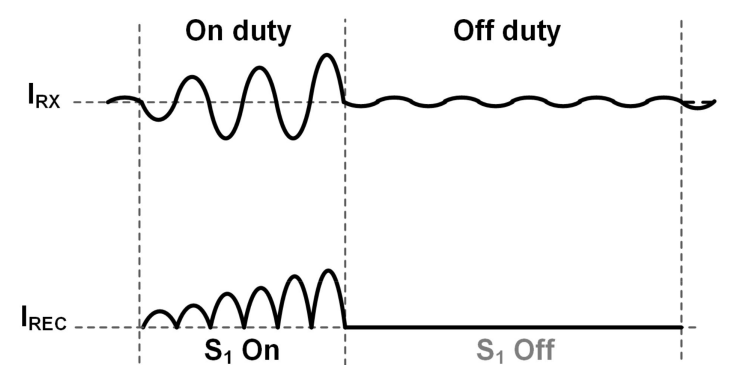

(b)

Figure 7. (a) The schematic of a resonant regulating rectifier (3R); and (b) the switching waveforms of $I_{\mathrm{RX}}$ and $I_{\mathrm{REC}}$, working in Discontinuous Conduction Mode (DCM) similar to a DC-DC converter [17].

To reduce the RMS resonant current, a three-switch 3R scheme working in CCM was also presented in [17]. The working principle of this scheme is shown in Figure 8. When in on duty, both $S_{1}$ and $S_{3}$ are turned on and $S_{2}$ is turned off, making $C_{\mathrm{FL}}$ and $C_{\mathrm{L}}$ connect in parallel. On the contrary, when in off duty, $S_{1}$ and $S_{3}$ are turned off and $S_{2}$ is turned on, resulting in $C_{\mathrm{FL}}$ and $C_{\mathrm{L}}$ being connected in series. Under this configuration, a significantly smaller equivalent resistance $R_{\text {eq }}$ can be achieved in off duty compared with DCM, allowing $I_{\text {REC }}$ to charge the load continuously similar to CCM as shown in Figure 8c. Similarly, the output voltage can be regulated using PWM to switch between the on- and off-duty states. However, considering DCM is still useful, especially under a light load condition, the three-switch $3 \mathrm{R}$ can also be configured to DCM by turning $S_{1}$ and $S_{3}$ on and $S_{2}$ off in the on state and turning $S_{1}, S_{1}$, and $S_{3}$ off in the off state. Hence, the load range can be extended by configuring the rectifier to DCM in light load conditions and CCM in heavy load conditions. This three-switch 3R showed a measured maximum 86\% PCE when the loading current ranged from 70 to $700 \mathrm{~mA}$, and precise regulation was achieved with a $3.3 \%$ output voltage deviation. 


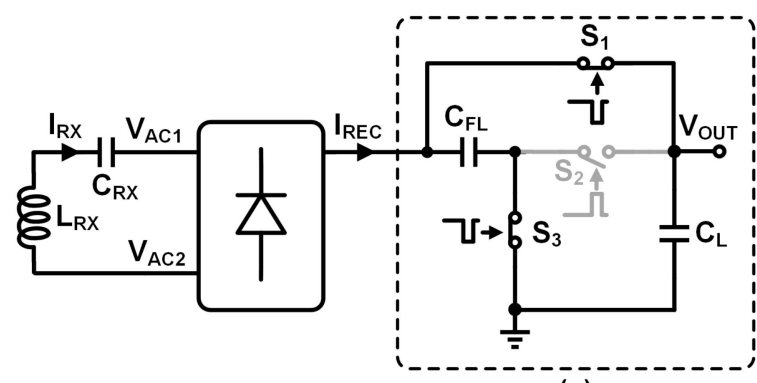

(a)

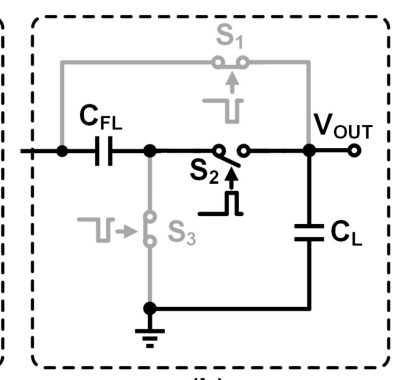

(b)

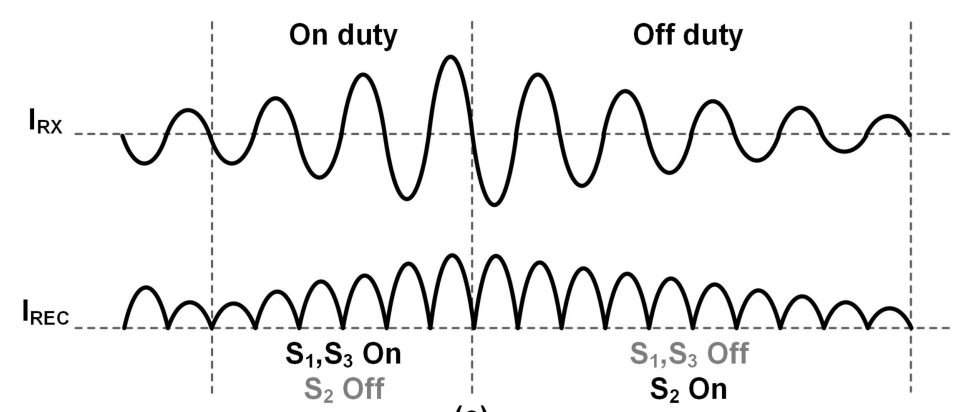

(c)

Figure 8. The schematic of the three-switch $3 \mathrm{R}$ in (a) an on-duty state when $S_{1}$ and $S_{3}$ are turned on and $S_{2}$ is turned off and (b) an off-duty state when $S_{1}$ and $S_{3}$ are turned off and $S_{2}$ is turned on. (c) Switching waveforms of $I_{\mathrm{RX}}$ and $I_{\mathrm{REC}}$ in Continuous Conduction Mode (CCM) [17].

Another issue for the $3 \mathrm{R}$ scheme is that a potentially high $V_{\mathrm{REC}}$ is expected at the beginning of off duty, which may damage the switches. To address this, a limiting capacitor $C_{\mathrm{LM}}$ is added between the rectifier inputs $\left(V_{\mathrm{AC} 1}\right.$ and $\left.V_{\mathrm{AC} 2}\right)$, which limits the $V_{\mathrm{REC}}$ to $V_{\mathrm{OUT}}+2 V_{\mathrm{D}}$ at on duty and $V_{\mathrm{LM}}-2 V_{\mathrm{D}}$ at off duty.

For the aforementioned post-stage regulations [12-20], they all suffer from loss from the cascaded stages. The PCE can be written as:

$$
\mathrm{PCE}=\eta_{\mathrm{rec}} \times \eta_{\text {reg }}
$$

where $\eta_{\text {rec }}$ and $\eta_{\text {reg }}$ are the efficiencies of the rectifier and post-stage regulators, respectively. As such, although $\eta_{\text {reg }}$ can be reasonably high, the PCE is still limited. Consequently, multiple one-stage regulation schemes have been proposed as a solution.

\subsection{One-Stage Regulation}

\subsubsection{Active Diode Conduction Time Control}

To eliminate the post regulator for a higher PCE, it is straightforward to integrate the rectification and regulation into one stage. The authors in [21-23] presented a one-stage regulating rectifier. In these schemes, the diode is implemented by a gate-controlled power transistor. The diode conduction time can be controlled by modulating the pulse width or the frequency of the power transistor gate control signal. As a result, the amount of current delivered to the load is controlled, and thus we have output regulation. These schemes are discussed as follows.

The authors in [21] proposed a regulating rectifier, and the regulation is achieved by controlling the active diode conduction time as in Figure 9a. The n-channel metal-oxide-semiconductor field-effect transistor (NMOS) $M_{1}$ and $M_{2}$ are cross-connected as the down rectifying diodes. For the up rectifying p-channel metal-oxide-semiconductor field-effect transistor (PMOS) $M_{3}$ and $M_{4}$, they are controlled by a pulse generator. When $V_{\mathrm{AC} 1}>V_{\mathrm{OUT}}, M_{3}$ is turned off and $M_{4}$ is turned on and vice versa. By controlling the $M_{3}$ and $M_{4}$ conduction time, $V_{\text {OUT }}$ regulation can be achieved. A PWM scheme will 
be straightforward, but the pulse width of the gate control signals $V_{\mathrm{GP} 1}$ and $V_{\mathrm{GP} 2}$ may be too narrow to turn on $M_{3}$ and $M_{4}$ under a light load condition for a high working frequency. To circumvent this, hybrid pulse modulation (HPM) is utilized as a combination of PWM and pulse frequency modulation (PFM) to provide regulation for a wide load range as given in Figure $9 \mathrm{~b}$. When the $V_{\mathrm{GP}}$ and $V_{\mathrm{GP} 2}$ pulse width controlled by PWM is decreased to a threshold value, PFM takes over the control loop by adjusting the $V_{\mathrm{GP} 1}$ and $V_{\mathrm{GP} 2}$ pulse frequency. The measurement result of [19] showed a maximum $60 \%$ PCE with a 10-mm coil distance. Obviously, the PCE is not optimized, which is mainly due to the relatively large rectifying diode drop (between $V_{\mathrm{AC} 1(2)}$ and $\left.V_{\mathrm{OUT}}\right)$ under certain loading conditions, especially when $M_{3}$ and $M_{4}$ may be turned on around the peak of $V_{\mathrm{AC1}(2)}$ as in Figure 10a. As such, an additional power loss will be found on $M_{3}$ and $M_{4}$ and thus a PCE degradation.

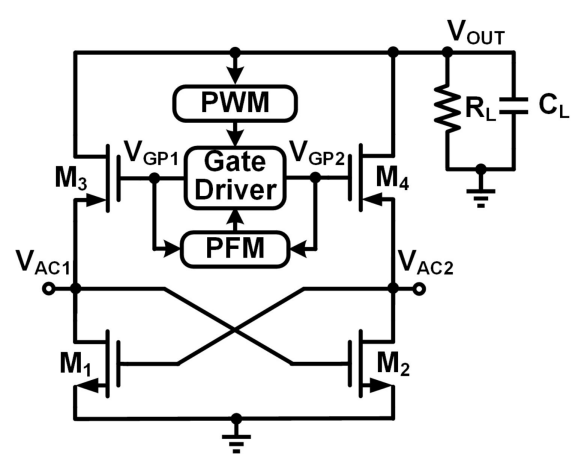

(a)

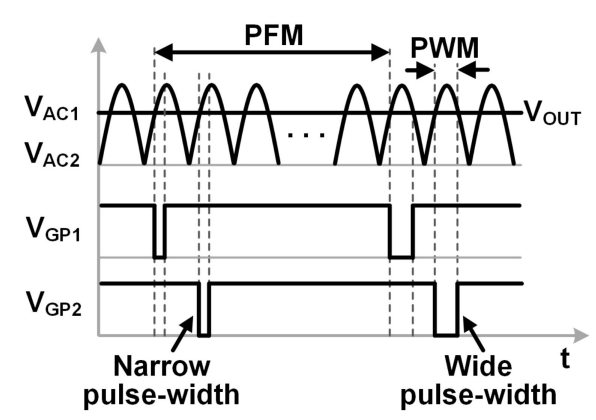

(b)

Figure 9. (a) The schematic of a regulating rectifier based on the hybrid pulse modulation (HPM) technique; (b) The waveforms of $V_{\mathrm{AC} 1}, V_{\mathrm{AC} 2}, V_{\mathrm{GP} 1}$, and $V_{\mathrm{GP} 2}$, where a narrow and wide pulse width is expected under light and heavy load conditions, respectively [21]. PFM, pulse frequency modulation; PWM, pulse width modulation.

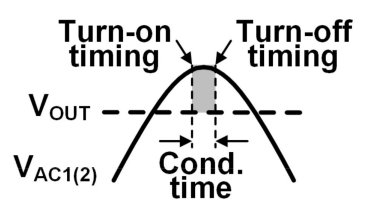

(a)

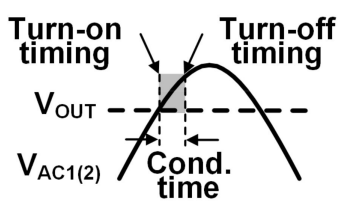

(b)

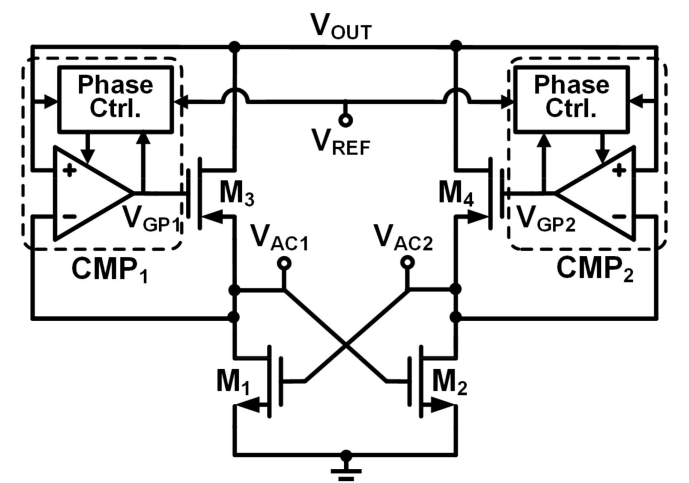

(c)

Figure 10. The conduction time starts at (a) the peak of $V_{\mathrm{AC} 1(2)}$; and (b) $V_{\mathrm{AC}}$ just exceeds $V_{\mathrm{OUT}}$; (c) The schematic of the regulating rectifier based on the turn-on phase control technique [22]. $\mathrm{CMP}$, comparator.

To address this issue, the authors of [22], whose idea is illustrated in Figure 10c proposed a turn-on phase control technique to turn the active diodes on with a minimized diode drop. The $M_{3}$ and $M_{4}$ gate control signals in this scheme are generated by the phase control comparator $\mathrm{CMP}_{1}$ and $\mathrm{CMP}_{2}$ rather than a conventional comparator. The waveforms of $V_{\mathrm{AC1}(2)}$ and $V_{\mathrm{OUT}}$ are illustrated in Figure 10b. When $V_{\text {AC }}$ just exceeds $V_{\text {OUT }}$, the rectifying diodes $M_{3}$ and $M_{4}$ are turned on, similar to a conventional rectifier, but they are turned off when $V_{\text {OUT }}$ exceeds a reference voltage $V_{\text {REF }}$. Moreover, $M_{3}$ and $M_{4}$ also adopt a dynamic body biasing circuit for further voltage drop reduction and PCE improvement. 
The measurement results showed that this scheme can provide a regulated $V_{\text {OUT }}$ from $2.5 \mathrm{~V}$ to $4.6 \mathrm{~V}$ with an improved PCE from $72 \%$ to $87 \%$.

The PCE can be even more improved by optimizing the conduction time of both the up and down rectifying diodes [23] as in Figure 11. The $V_{\text {OUT }}$ regulation is achieved by adaptively adjusting the duty-cycle $D$ and phase $\phi$ of the gate control signals $V_{\mathrm{GP} 1}, V_{\mathrm{GP} 2}, V_{\mathrm{GN} 1}$, and $V_{\mathrm{GN} 2}$ simultaneously with a rectification controller. The desired $D$ and $\phi$ are adaptively synthesized based on an $I_{R X}$ sensor using $M_{5}$ and $M_{6}$. The measurement result showed that this phase and duty-cycle control can regulate the output voltage from $3.5 \mathrm{~V}$ to $5 \mathrm{~V}$, and the maximum PCE was $96 \%$ at a $4.64 \mathrm{~V}$ output voltage.

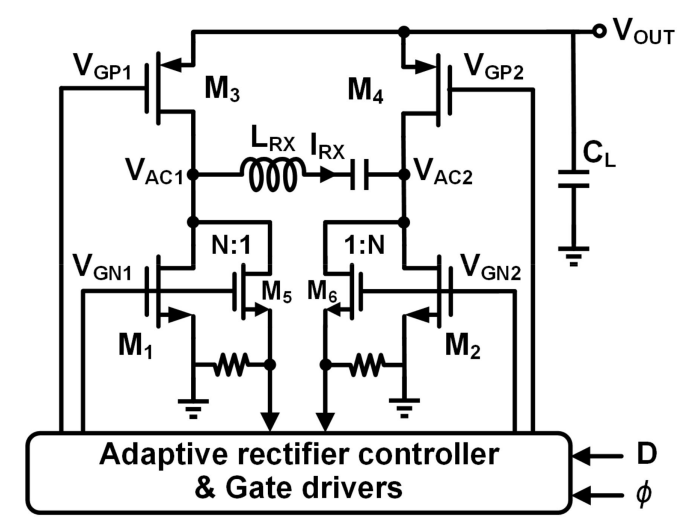

Figure 11. The schematic of the adaptive regulating rectifier based on phase and duty-cycle control [23].

\subsubsection{Reconfigurable Regulating Rectification}

Another one-stage topology is based on the reconfigurable resonant regulating $\left(\mathrm{R}^{3}\right)$ rectification technique $[24,25]$. In these schemes, the $\mathrm{R}^{3}$ rectifier can be reconfigured to multiple basic rectifier topologies, e.g., full-wave $(1 \times)$ and half-wave rectifiers $\left(\frac{1}{2} \times\right)$ and a voltage doubler $(2 \times)$, and the rectifier output voltages are $1 \times, \frac{1}{2} \times$, and $2 \times V_{\text {OUT, }}$, respectively. As such, $\mathrm{RX}$ regulation can be achieved by switching among these topologies.

In [26,27], a $1 \times / 2 \times$ reconfigurable rectifier was demonstrated as in Figure 12, where the switches $S_{1 \sim 5}$ are utilized to fulfill the reconfiguration. When $S_{3}, S_{4}$ is turned on while the others are turned off, the power transistors $M_{3}$ and $M_{4}$ are cross-connected as two upper diodes, and $D_{1}$ and $D_{2}$ act as active diodes. In this scenario, it works as a full-wave rectifier as shown in Figure 12a. On the other hand, when $S_{1}$ and $S_{2}$ are turned on while $S_{3}$ and $S_{4}$ are turned off, $M_{1}$ and $M_{3}$ are configured to active diodes and $M_{2}$ and $M_{4}$ are disabled. Meanwhile, $S_{5}$ is turned on to connect $V_{\mathrm{AC} 2}$ to the middle of the two load capacitors $C_{\mathrm{L} 1}$ and $C_{\mathrm{L} 2}$, making the rectifier a voltage doubler as depicted in Figure 12b. It should be noted that $R_{\text {eq }}$ equals $R_{\mathrm{L}} / 2$ and $R_{\mathrm{L}} / 8$ in $1 \times$ and $2 \times$ mode, respectively.

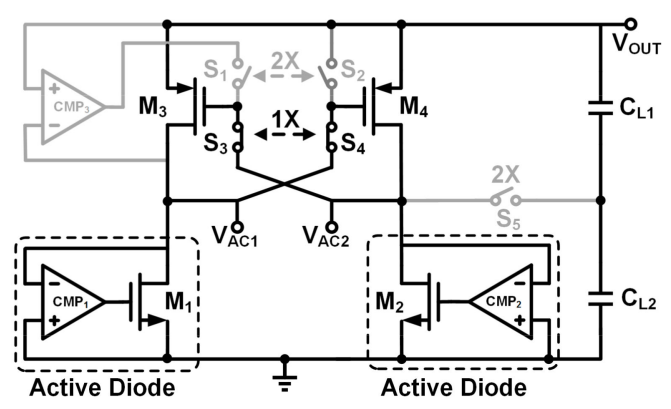

(a)

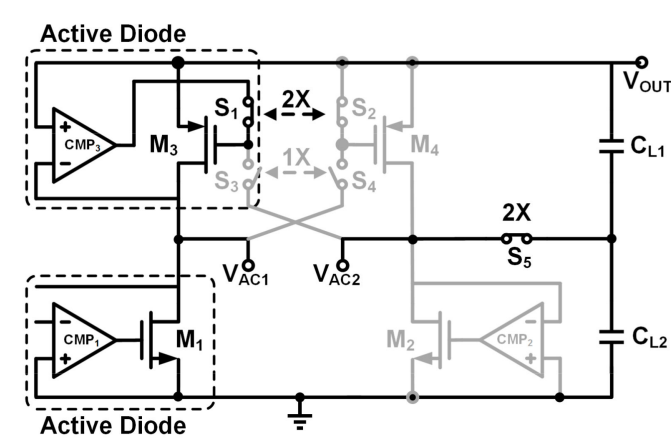

(b)

Figure 12. $1 \times / 2 \times$ reconfigurable rectifier operates $(\mathbf{a})$ in $1 \times$ mode; and (b) in $2 \times$ mode [26], active diodes are composed of the gate-controlled power transistor and comparator. 
This reconfigurable rectifier topology was applied in [24] for regulation with PWM control as illustrated in Figure 13a. Once $V_{\mathrm{AC} 1(2)}$ has a fixed amplitude, the rectifier will output voltages $V_{1 \times}$ and $V_{2 \times}$ at $1 \times$ and $2 \times$ mode, respectively. As shown in Figure 13b, the PWM controller switches the reconfigurable rectifier periodically between the two modes so that $V_{\text {OUT }}$ can be regulated at an intermediate voltage between $V_{1 \times}$ and $V_{2 \times}$. Thanks to the one-stage scheme, the maximum PCE of this $\mathrm{R}^{3}$ rectifier was $92.6 \%$ at a $60 \mathrm{~mW}$ output power in [24].

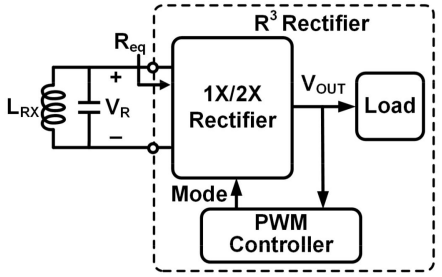

(a)

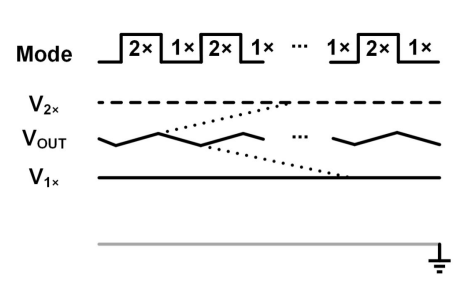

(b)

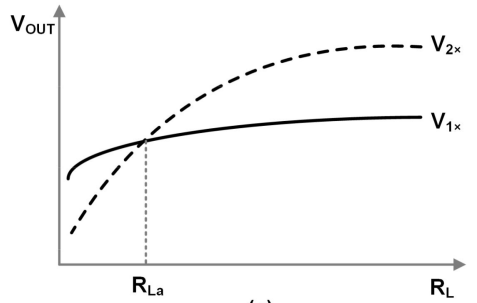

(c)

Figure 13. (a) The block diagram of the reconfigurable resonant regulating $\left(\mathrm{R}^{3}\right)$ rectifier; $(\mathrm{b})$ the PWM regulation principle; and (c) $V_{1 \times}$ and $V_{2 \times}$ versus load resistance, where the $R_{\mathrm{La}}$ is the breakeven value and at which $V_{2 \times}=V_{1 \times}[24]$.

However, the $\mathrm{R}^{3}$ rectifier in [24] may fail to correctly regulate $V_{\text {OUT }}$ under certain circumstances. Firstly, $V_{\text {OUT }}$ cannot be regulated to lower than $V_{1 \times}$ or higher than $V_{2 \times}$. Secondly, $V_{\text {OUT }}$ cannot be correctly regulated if the term $V_{2 \times}>V_{1 \times}$ is not true, which is discussed as follows.

From [43], $V_{2 \times}$ and $V_{1 \times}$ can be written as:

$$
\begin{aligned}
& V_{1 \mathrm{X}}=k \sqrt{\frac{L_{\mathrm{RX}}}{L_{\mathrm{TX}}}} \frac{Q_{1} Q_{2}}{1+k^{2} Q_{1} Q_{2}+Q_{2} / Q_{\text {load }, 1 \times}} \cdot A_{1 \times} \cdot V_{\mathrm{S}} \\
& V_{2 \mathrm{X}}=k \sqrt{\frac{L_{\mathrm{RX}}}{L_{\mathrm{TX}}}} \frac{Q_{1} Q_{2}}{1+k^{2} Q_{1} Q_{2}+Q_{2} / Q_{\text {load }, 2 \times}} \cdot A_{2 \times} \cdot V_{\mathrm{S}}
\end{aligned}
$$

where $A$ is the voltage conversion ratio $V_{\mathrm{OUT}} / V_{\mathrm{AC}}$. From Equations (5) and (6), there exists a breakeven value where $V_{2 \times}=V_{1 \times}$, which can be given by:

$$
R_{\mathrm{La}}=\frac{2 Q_{2}}{\omega_{\text {res }} C_{2}\left(1+k^{2} Q_{1} Q_{2}\right)} \cdot \frac{A_{1 \times} A_{2 \times}}{A_{2 \times}-A_{1 \times}}
$$

and the resulting $V_{1 \times}$ and $V_{2 \times}$ versus the load is presented in Figure 13c. As can be seen, $V_{2 \times}>V_{1 \times}$ will possibly take place under some heavy loading condition $\left(R_{\mathrm{L}}<R_{\mathrm{La}}\right)$, which undermines the regulation.

To overcome the limited load range and facilitate correct regulation, a three-mode $R^{3}$ rectifier is presented in [25]. As illustrated in Figure 14, it can be configured into three modes: a full-bridge rectifier $\left(1 \times\right.$ mode), a half-bridge rectifier $\left(\frac{1}{2} \times\right.$ mode $)$, and freewheeling $\left(0 \times\right.$ mode). The $\frac{1}{2} \times$ mode is added to fulfill an even distribution of power for a reduced output voltage ripples. The output regulation of this rectifier can be achieved by switching between two of the three modes. Specifically, it switches periodically between $1 \times$ and $\frac{1}{2} \times$ mode under a heavy load, and between $\frac{1}{2} \times$ and $0 \times$ mode under a light load. These two control schemes can be automatically changed based on the output current sensed. A first and Type II compensation are adopted in PWM to obtain a fast response and a stabilized system. A peak $92.2 \%$ PCE was measured with a $3.5 \mathrm{~W}$ output power, and a maximum $6 \mathrm{~W}$ output power was observed.

To sum up, the aforementioned regulating rectifiers [21-27] can achieve high PCE and self-regulation. However, the VCE is typically low in these schemes, which is not favorable especially when the coil distance is increased or the coils are misaligned. 


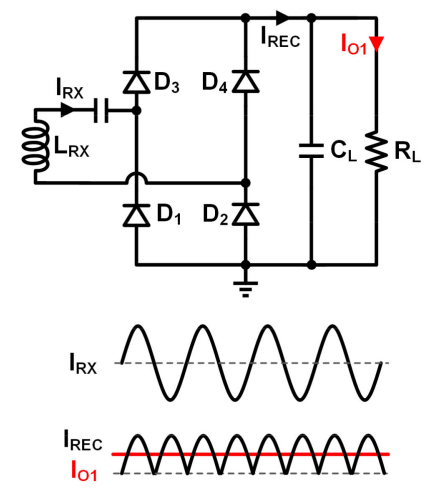

(a)
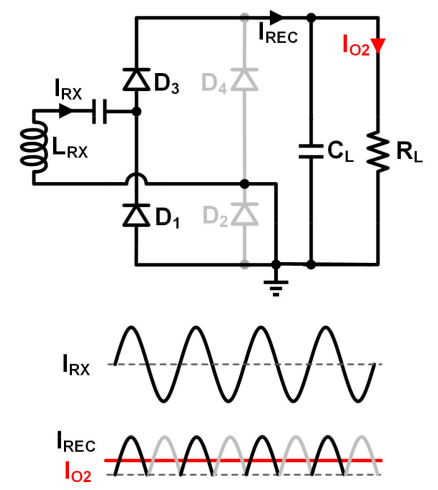

(b)
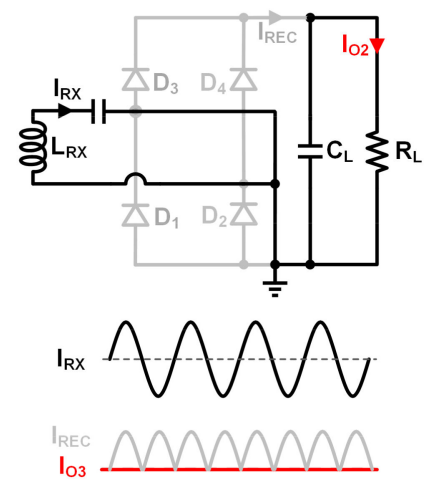

(c)

Figure 14. The three-mode $\mathrm{R}^{3}$ rectifier configured as (a) a full-bridge rectifier ( $1 \times$ mode);

(b) a half-bridge rectifier $\left(\frac{1}{2} \times\right.$ mode $)$; and (c) freewheeling $(0 \times$ mode $)$ [25].

\subsubsection{Switching-Based Current-Mode Regulation}

To achieve a high VCE, a switching-based current-mode (SCM) regulating technique was proposed in [28-31]. This technique differs from voltage-mode ones by firstly storing the received energy in the RX LC resonant tank (as the resonant phase) and then delivering it to the load (charging phase). With SCM regulation, the power delivered to the load (PDL) can be regulated by controlling the length of the resonant phase.

A SCM RX based on Q-modulation was proposed in [28] as in Figure 15a, where the regulation is realized by modulating $Q_{\text {load }}$. As in Figure $15 \mathrm{~b}$, the resonant phase (with $T_{\Phi 1}$ duration) is activated by turning on the single switch $S_{1}$. For maximum energy storing, $S_{1}$ is turned on at the zero-crossing point of the resonant current $I_{R X}$, as in Figure 16a, which should be twice every cycle. Additionally, when SW is turned off, it is in a charging phase (with $T_{\Phi 2}$ duration) as in Figure 15c.

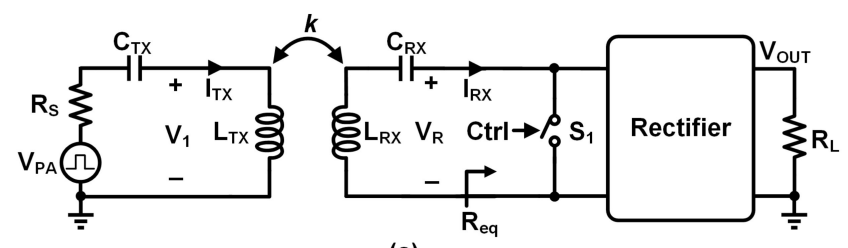

(a)

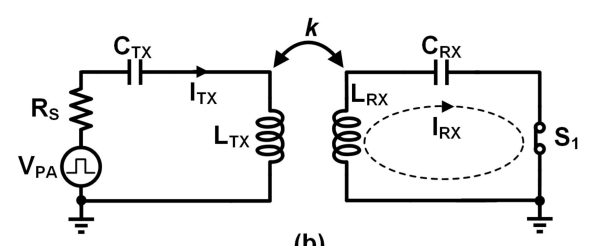

(b)

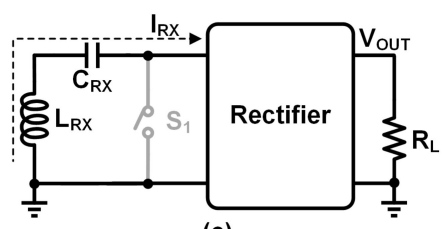

(c)

Figure 15. (a) The block diagram of the switching-based current-mode (SCM) RX based on Q-modulation, which works at (b) the resonant phase and (c) the charging phase [28].

Two advantages can be found in this SCM RX. For one thing, the equivalent resistance $R_{\mathrm{eq}}$ and thus the Q-factor are modulated by the duration ratio $\left(m=T_{\Phi 1} / T_{\Phi 2}\right)$ as

$$
R_{\mathrm{eq}}=R_{\mathrm{L}}\left[1-D+\frac{1}{2 \pi} \sin (2 \pi D)\right]
$$


where $D=m /(1+m)$. Consequently, by adjusting $m$, the received and thus output power can be regulated. For the other thing, according to Equation (8), the equivalent resistance $R_{\mathrm{eq}}$ is decreased in Q-modulation, and thus the $Q_{\text {load }}$ is increased. The $V_{\mathrm{R}}$ in this case can be expressed as:

$$
V_{\mathrm{R}}=k \sqrt{\frac{L_{\mathrm{RX}}}{L_{\mathrm{TX}}}} \frac{Q_{1} Q_{2}}{1+k^{2} Q_{1} Q_{2}+Q_{2} / Q_{\mathrm{load}}} \cdot V_{\mathrm{S}}
$$

indicating that $V_{\mathrm{R}}$ can be boosted without using a bulky inductor as in a conventional boost DC-DC converter. The measured results showed that the $V_{\mathrm{R}}$ can be boosted to $5.5 \mathrm{~V}$.

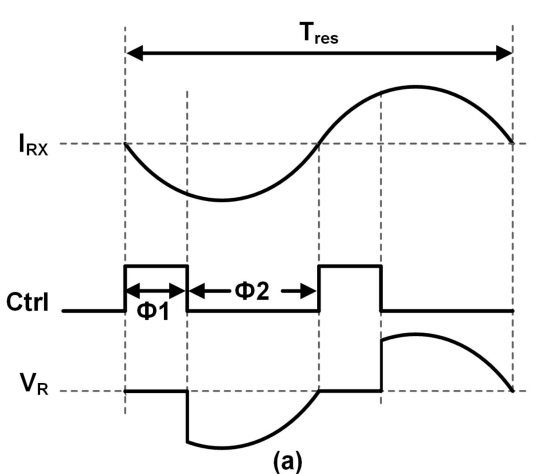

(a)

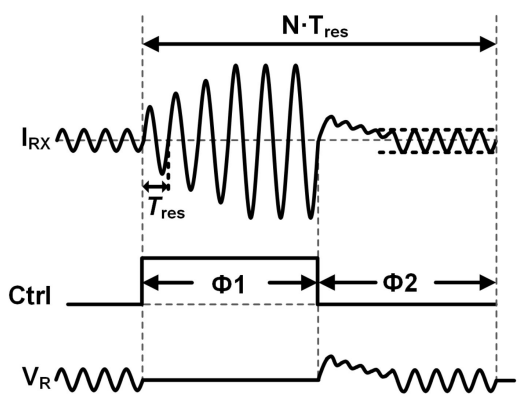

(b)

Figure 16. The switching waveforms of (a) Q-modulation [28] and (b) multi-cycle Q-modulation (MCQ) [30]. Ctrl is on-off control signal of the switch $S_{1}$.

However, when maximum energy is stored in the $L_{R X} C_{R X}$ tank, the SW turn-on timing should be restrictively synchronized to the $I_{\mathrm{RX}}$ zero-crossing point, which significantly increases the design difficulty. In addition, this synchronization makes it difficult for the system to work at a high resonant frequency, e.g., the $2 \mathrm{MHz}$ resonant frequency in [28]. The measured result showed that the PTE was $40.5 \%$ at $R_{\mathrm{L}}=200 \Omega$ with an 8 -cm coil distance, and greater than $20 \%$ PTE can be achieved when $R_{\mathrm{L}}$ ranges from 50 to $1000 \Omega$.

To allow for a higher resonant frequency, a multi-cycle Q-modulation (MCQ) technique was introduced in [30] that works at a $13.56 \mathrm{MHz}$ resonant frequency. The principle of MCQ is similar to that of [28], but both the resonant and charging phase are extended to multiple carrier cycles as in Figure 16b. MCQ can work without synchronizing the SW turn-on instant to the $I_{\mathrm{Rx}}$ zero-crossing points, which reduces the design difficulty. To overcome resonant frequency shifting due to the parasitic capacitance in the wireless link, an automatic resonance tuning scheme is also introduced in [30]. The measured result showed a $28.02 \%$ PTE at $R_{\mathrm{L}}=100 \Omega$. Compared with a voltage-mode rectifier, the $V_{\text {OUT }}$ has been increased significantly from an average of 2.61 to $4.15 \mathrm{~V}$ with the same input, coupling, and loading conditions.

In sum, the SCM technique can achieve a high $V_{\mathrm{R}}$ without a voltage doubler or boost converter. This is due to the fact that the high- $Q$ resonant tank is established by reducing the load in the resonant phase such that the $V_{\mathrm{R}}$ can be much higher, resulting in a high output voltage delivered to the load. In addition, output regulation can be achieved by modulating $R_{\text {eq }}$. Unfortunately, in many cases PDL regulation and PTE optimization cannot be simultaneously achieved.

Table 1 presents a comparison of the $\mathrm{RX}$ regulation topologies used in state-of-the-art works. For the post-stage regulation, although they have a high rectifier efficiency, PCEs are limited due to the loss from the cascaded stage. By contrast, the one-stage schemes typically achieve a higher PCE, except [19] where a large rectifying diode drop takes place. In addition, to address the low VCE of the regulating rectifiers [21-25], the SCM technique is proposed in [28,31] to boost the $V_{\mathrm{R}}$. The VCE of [28] is still smaller than 1 due to its application to $R_{\mathrm{L}}$ in the range of hundreds of ohms and below, while it is much higher in [31] when applied to a low output power system. 
Table 1. The comparison of RX regulation topologies in state-of-the-art R-WPT systems.

\begin{tabular}{|c|c|c|c|c|c|c|c|c|c|c|}
\hline References & [12] & [16] & [17] & [21] & [22] & [23] & [24] & [25] & [28] & [31] \\
\hline Topology & \multicolumn{3}{|c|}{ Post-stage regulation } & \multicolumn{7}{|c|}{ One-stage regulation } \\
\hline Max. PDL & $40 \mathrm{~mW}$ & $6 \mathrm{~W}$ & $6 \mathrm{~W}$ & $0.7 \mathrm{~mW}$ & $12.8 \mathrm{~mW}$ & $2.68 \mathrm{~W}$ & $0.1 \mathrm{~W}$ & $6 \mathrm{~W}$ & $1.45 \mathrm{~W}$ & $96 \mu \mathrm{W} \#$ \\
\hline Max. Rectifier Eff. & $85 \%$ & $92.8 \%$ & $92.5 \% \#$ & \multicolumn{7}{|c|}{ Rectifier efficiency $=$ PCE for one-stage regulation } \\
\hline Max. PCE & $74.8 \%$ & $84.6 \%$ & $86 \%$ & $60 \%$ & $87 \%$ & $96 \%$ & $92.6 \%$ & $92.2 \%$ & $87.1 \%$ & N/A \\
\hline Max. PTE & N/A & N/A & $55 \%$ & N/A & $\mathrm{N} / \mathrm{A}$ & $47.2 \%$ & $50 \%$ & N/A & $\sim 46 \%{ }^{\#}$ & $5.3 \%$ \\
\hline Max. VCE & N/A & N/A & N/A & 0.92 & 0.92 & $\mathrm{~N} / \mathrm{A}$ & N/A & $\sim 0.9^{\#}$ & $0.82 \#$ & 3.1 \\
\hline
\end{tabular}

\# Calculated from figures in the paper. PDL, power delivered to the load; PCE, power conversion efficiency; PTE, power transmission efficiency; VCE, voltage conversion efficiency.

\section{TX Regulation}

Besides regulation at the RX side, the output can be regulated at the TX side as well. This scheme is defined as TX regulation, which can be achieved by controlling the PA supply voltage [24,32,33], the resonant frequency [34], or the power switch duty cycle [36-40]. The necessity for TX regulation and its implementations are discussed in the following sections.

\subsection{Why TX Regulation is Necessary}

TX regulation is pivotal in many cases. For one thing, TX regulation manages to widen the load range considering that the $\mathrm{RX}$ regulation range is typically limited. For the other thing, it is difficult to simultaneously optimize the PTE with RX regulation under a wide range of coil coupling distances, misalignments, or loading conditions. Hence, to optimize the PTE, TX regulation based on global feedback from the RX side is necessary.

The authors in [24] presented a scenario for how TX regulation is useful to extend the output range, where $V_{2 \times}<V_{1 \times}$ and thus false regulation may take place under certain load conditions. To handle this, the TX output power was increased during the $2 \times$ mode to ensure that $V_{2 \times}>V_{1 \times}$ was unconditionally correct. The load condition at the RX side can be fed back to the TX side to facilitate TX power control. As shown in Figure 17a, the feedback is achieved with a global control loop, and the load condition can be sensed by a backscattering technique. Then, the significant difference of $R_{\text {eq }}$ between $1 \times$ and $2 \times$ mode is backscattered to the primary side, causing an $I_{T X}$ difference, which is sensed by an additional coil $L_{\text {sen }}$ coupling to the TX coil. This additional coil only takes up a small area so as to minimize its effect on the coupling coefficient and the cost overhead.

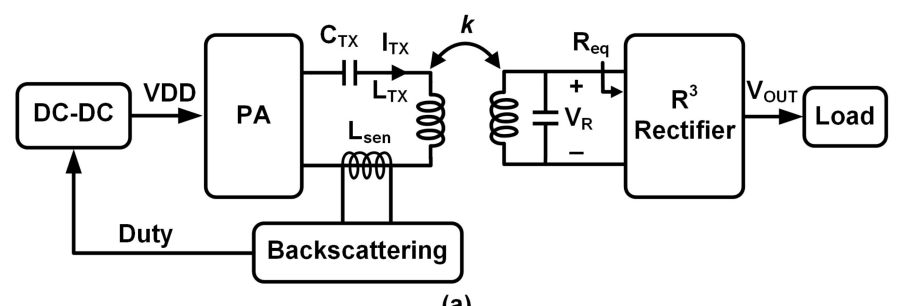

(a)

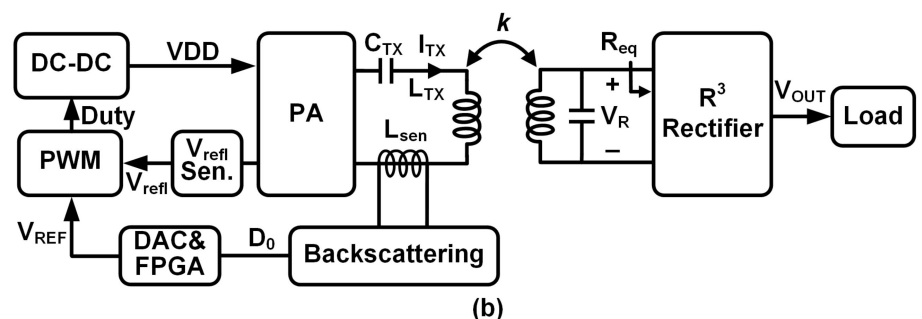

Figure 17. The block diagram of TX regulation is based on (a) supply voltage control [24] and (b) $V_{\text {refl }}$ control [32] for extending the regulation range. PA, power amplifier. 
The authors in [32] demonstrated another TX regulation scheme to ensure $V_{2 \times}>V_{1 \times}$. Based on the analysis in [32], the $V_{\mathrm{R}}$ is approximately proportional to $V_{\text {refl, }}$ which means that $V_{\mathrm{R}}$ can be indirectly regulated by regulating $V_{\text {refl }}$ at the TX side instead. In this scheme, a fixed $V_{\mathrm{R}}$ can be achieved over a much wider workable coupling and loading range. Within this range, $V_{2 \times}$ is approximately twice $V_{1 \times}$, leading to correct output voltage regulation.

To regulate $V_{\text {refl }}$, it must be sensed first. Unfortunately, $R_{\text {refl }}$ is not a physical resistor, so $V_{\text {refl }}$ cannot be sensed directly. Hence, as illustrated in Figure $17 \mathrm{~b}$, a current-based $V_{\text {refl }}$ sensor is applied for $V_{\text {refl }}$ detection. Then, by adjusting the supply voltage using a PWM-controlled DC-DC converter, the $V_{\text {refl }}$ can be regulated to be equal to reference voltage $V_{\mathrm{REF}}$ under different modes in an $\mathrm{R}^{3}$ rectifier, keeping $V_{\mathrm{R}}$ constant. In addition, the mode switching information $D_{0}$ can feed back by backscattering and then convert into $V_{\text {REF }}$ by DAC (digital to analog converter) and FPGA (field programmable gate array). Measurement results showed that with this TX regulation, the workable coupling and loading ranges were extended by $250 \%$ with $120 \mathrm{~mW}$ output power and by $300 \%$ with a $1.2-\mathrm{cm}$ coil distance compared to [24]. In addition, a maximum 92.5\% PCE and 62.4\% PTE were achieved.

\subsection{Power Amplifier Supply Voltage Control}

The PA supply voltage control, which is typically achieved with a DC-DC converter [33], is the most straightforward approach to TX regulation. Let us take a TX based on a class D PA as an example. As shown in Figure 18a, by setting a proper $V_{\mathrm{G} 1}$ and $V_{\mathrm{G} 2}$ to drive the $M_{1}$ and $M_{2}$, the PA will output a square wave $V_{\mathrm{PA}}$ with a $50 \%$ duty cycle as in Figure $18 \mathrm{~b}$, and the Fourier series of $V_{\mathrm{PA}}$ can be expressed as:

$$
V_{\mathrm{PA}}(\mathfrak{t})=\frac{V D D}{2}+\frac{2 V D D}{\pi}\left(\sin \left(\omega_{s w} t\right)+\frac{1}{3} \sin \left(3 \omega_{s w} t\right)+\frac{1}{5} \sin \left(5 \omega_{s w} t\right)+\cdots+\frac{1}{n} \sin \left(n \omega_{s w} t\right)+\cdots\right)
$$

where $\omega_{\mathrm{sw}}=2 \pi f_{\mathrm{sw}}$ and $f_{\mathrm{sw}}$ is the switching frequency. It is noteworthy that here $n$ is an odd number. Then, the DC and high order harmonics components are filtered out by the $L_{\mathrm{TX}} C_{\mathrm{TX}}$ tank. Consequently, the PA output voltage $V_{\mathrm{PA}}$ is proportional to the supply voltage from Equation (10), and thus $V_{\mathrm{PA}}$ can be regulated by controlling the supply voltage. Measurement results in [33] showed that a $40.2 \%$ PTE improvement had been achieved with a 7-mm coil distance and $250 \mathrm{~mW}$ output power.

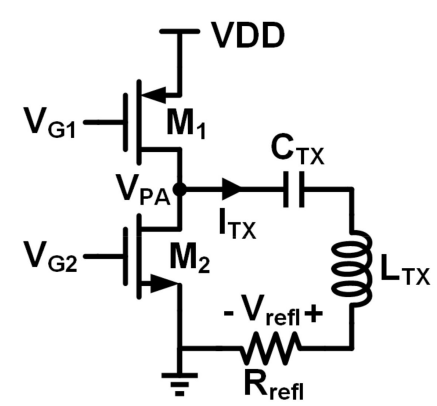

(a)

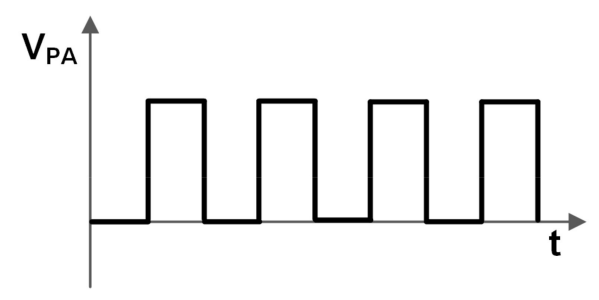

(b)

Figure 18. The schematic of a class-D PA.

\subsection{Resonant Frequency Control}

It should be noted that TX regulation can be achieved not only by adjusting the supply voltage, but also the resonant frequency $f_{\text {res }}$. The more $f_{\text {res }}$ is tuned to be deviated from the resonant frequency of the $L_{T X} C_{T X}$ tank, the less power will be transmitted.

The authors in [34] presented a resonant frequency control scheme as illustrated in Figure 19. The resonant frequency here was adjusted by controlling the value of the capacitor $C_{\mathrm{TX}}$ in the resonant TX tank. The $C_{\mathrm{TX}}$ implements a weighted-capacitor array, which is controlled dynamically by the load condition from the RX side through a wireless communication network. The measurement results 
showed that the PDL can be regulated effectively over a wide load range, and $80 \%$ PCE can be achieved when transferring $15 \mathrm{~W}$ over a $10-\mathrm{mm}$ coil distance.

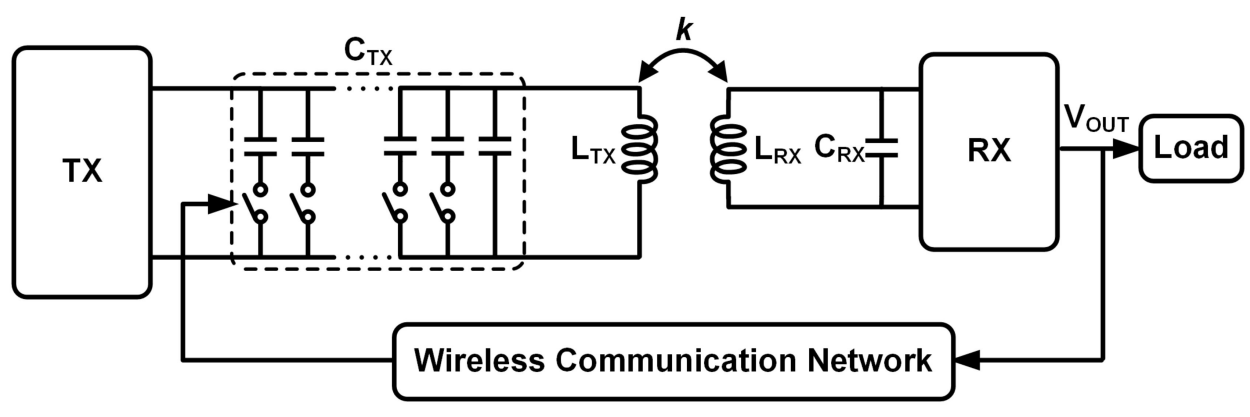

Figure 19. TX regulation based on resonant frequency control, which is achieved with a weighted-capacitor array [34].

Nonetheless, the $C_{\mathrm{TX}}$ in [34] can only be tuned discretely, which prevents high-resolution regulation. Furthermore, for the commonly used megahertz Industrial Scientific Medical (ISM) band, the allowable bandwidth is pretty narrow, e.g., $15 \mathrm{KHz}$ for $6.78 \mathrm{MHz}$ and $7 \mathrm{KHz}$ for $13.56 \mathrm{MHz}$ [44]. Consequently, the tunable $f_{\text {res }}$ and regulation range will be limited to accommodate the ISM specifications. Additionally, a low PTE is expected, since the resonant frequency of the TX LC tank is purposely adjusted to deviate from the RX tank's resonant frequency for regulation [43].

\subsection{PA Power Switch Duty-Cycle Control}

TX regulation can also be implemented by a PA power switch duty-cycle control technique based on switching frequency modulation [35,36], pulse density modulation [37,38], and phase shifted modulation $[39,40]$. These schemes advance the resonant frequency control scheme by keeping a constant $f_{\text {res. }}$. These schemes are discussed in the following sections.

\subsubsection{Switching Frequency Modulation}

It should be noted that $f_{\mathrm{sw}}$ can be also designed to not be equal to $f_{\text {res }}$. According to Equation (10), when $f_{\text {sw }}=f_{\text {res }} / n$, the $n$th subharmonic component can be used for the power transmission. Considering that the DC and other components are filtered out by the TX resonant tank, $V_{\text {refl }}$ can be written as:

$$
V_{\text {refl }}(\mathrm{t})=\frac{1}{n} \times \frac{2 V D D}{\pi} \times\left.\sin \left(n \omega_{\mathrm{sw}} t\right)\right|_{f_{\mathrm{sw}}=\frac{1}{n} f_{\text {res }}}=\frac{f_{\mathrm{sw}}}{f_{\text {res }}} \times \frac{2 V D D}{\pi} .
$$

According to Equation (11), $V_{\text {refl }}$ is proportional to $f_{\text {sw }}$. Therefore, changing $f_{\text {sw }}$ according to the global feedback manages to regulate $V_{\text {refl }}$ and thus $V_{\text {OUT }}$.

The authors in [35] demonstrated a switching frequency modulation technique as illustrated in Figure 20. In this work, the PA switching frequency $f_{\mathrm{sw}}$ can be set to $f_{\text {res }}$ or $f_{\text {res }} / 3$ as shown in Figure 21a,b, respectively. Based on Equation (11), the $V_{\text {refl }}$ under $f_{\mathrm{sw}}=f_{\text {res }} / 3$ is $1 / 3$ of that when $f_{\text {sw }}=f_{\text {res }}$. Hence, $V_{\text {refl }}$ can be regulated by switching $f_{\text {sw }}$ between $f_{\text {res }}$ and $f_{\text {res }} / 3$ using a PWM scheme while the resonant frequency is kept constant. Additionally, the error amplifier (EA) output is fed back to TX through the wired data link, and a 2nd $\Delta-\Sigma$ modulator is applied to switch the PA between the $f_{\text {res }}$ and $f_{\text {res }} / 3$ modes to reduce spurious emission. However, the regulation range is limited from $f_{\text {res }} / 3$ to $f_{\text {res. }}$. The measured results showed that maximum power of $0.52 \mathrm{~W}$ can be transferred with a $50 \%$ PTE.

To extend the regulation range, [36] presented an enhanced subharmonic resonant switching scheme. Different from [35], the regulation can be achieved not only using odd-order harmonics, but also even-order harmonics. An even-order harmonic is implemented by averaging the adjacent 
two odd-order harmonics in the time domain, e.g., $f_{\text {res }} / 4$ can be achieved by averaging out $f_{\text {res }} / 3$ and $f_{\text {res }} / 5$ as illustrated in Figure 22b. As such, any frequency within $\left(0, f_{\text {res }}\right]$ can be chosen as $f_{\mathrm{sw}}$, resulting in a wider $V_{\text {OUT }}$ regulation range. The measurement results showed a $50 \% V_{\text {OUT }}$ regulation range extension with the proposed scheme compared to [35].

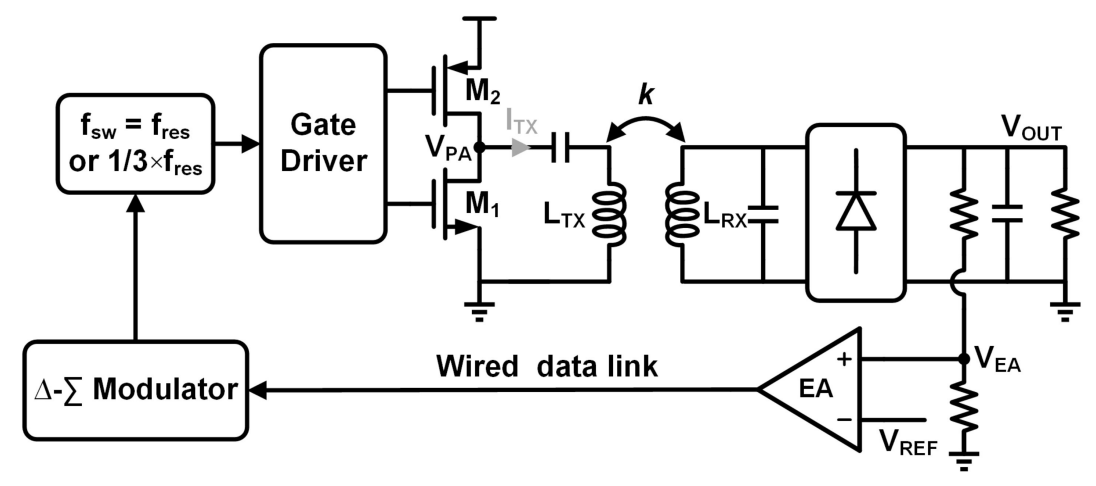

Figure 20. The block diagram for subharmonic resonant switching [35]. EA, error amplifier.

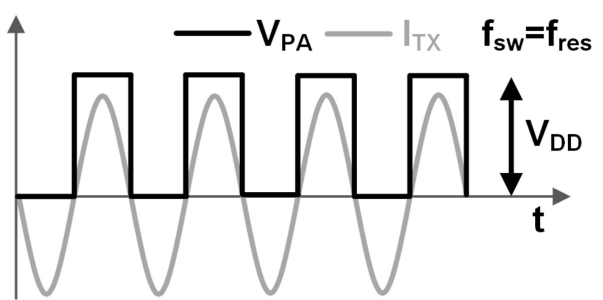

(a)

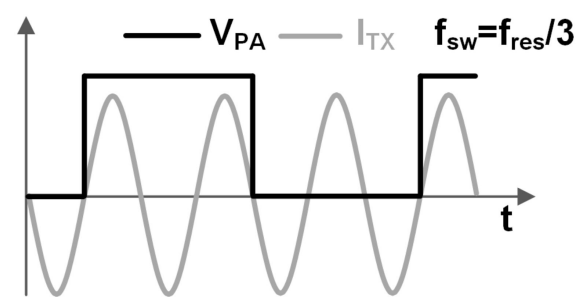

(b)

Figure 21. The PA $V_{\mathrm{PA}}$ and $I_{\mathrm{TX}}$ waveforms when $f_{\mathrm{sw}}=(\mathbf{a}) f_{\text {res }}$ and $(\mathbf{b}) f_{\text {res }} / 3$ [35].

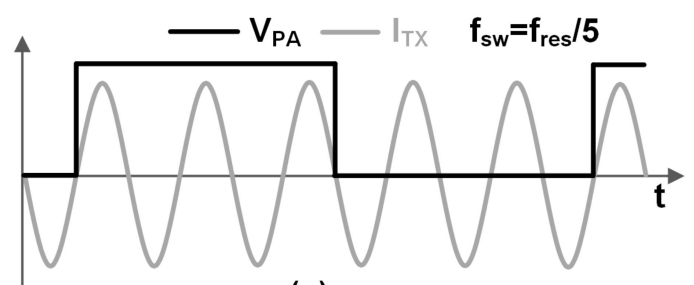

(a)

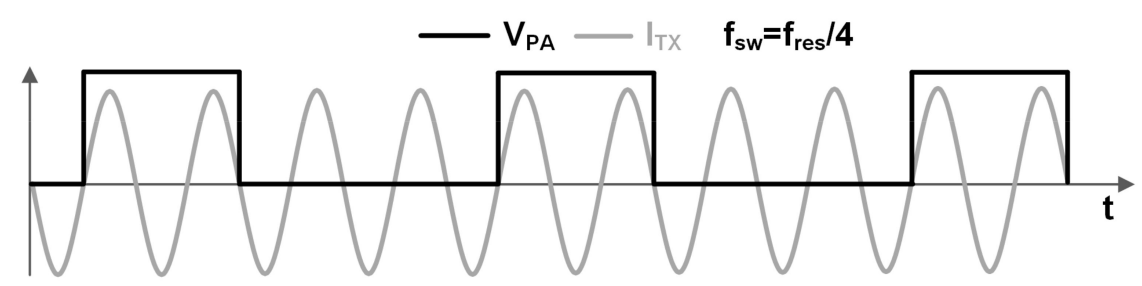

(b)

Figure 22. The PA $V_{\mathrm{PA}}$ and $I_{\mathrm{TX}}$ waveforms when $f_{\mathrm{sw}}=(\mathbf{a}) f_{\text {res }} / 5$ and $(\mathbf{b}) f_{\text {res }} / 4$ by averaging out $f_{\text {res }} / 3$ and $f_{\text {res }} / 5[36]$.

\subsubsection{Pulse Density Modulation}

The authors in [37] showed a pulse density modulation (PDM) technique based on On-Off Keying (OOK) modulation. This scheme regulates the transmitted power by switching the PA between the working and idle modes using OOK modulation. As illustrated in Figure 23, the differential PA 
consists of four power switches $M_{1 \sim 4}$, which are driven by the gate control voltages $\left(V_{\mathrm{G} 1 \sim 4}\right)$ with the resonant frequency. The switching signal $S_{1}$, modulated with an OOK envelope $S_{2}$, are applied to generate the gate control signals $V_{\mathrm{G} 1 \sim 4}$. When $S_{2}$ is logic high, the PA operates normally and when $V_{\mathrm{LF}}$ is logic low, the PA operates in idle mode. The measured maximum PTE was $72.6 \%$ at a $10 \Omega$ load resistance with a $0.2-\mathrm{m}$ coil distance.

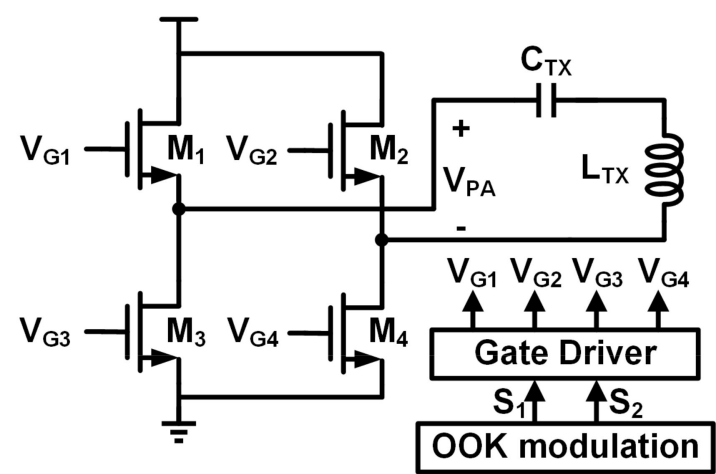

(a)

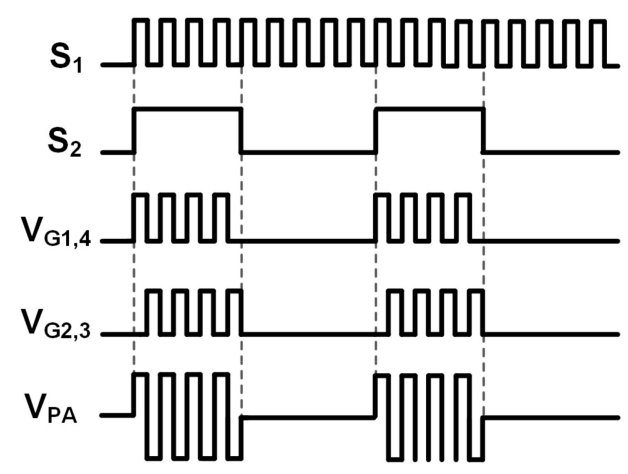

(b)

Figure 23. (a) The schematic of TX regulation using PDM based on On-Off Keying (OOK) modulation, and (b) the waveforms of $S_{1}, S_{2}, V_{\mathrm{G} 1 \sim 4}$, and $V_{\mathrm{PA}}$ [37].

The authors in [35] proposed another PDM technique for both differential PA output voltage $V_{\mathrm{PA}}$ regulation and optimized efficiency as illustrated in Figure 24a. This scheme regulates $V_{\mathrm{PA}}$ by adjusting the pulse density $d$, which is defined as the total density of the positive (P) and negative $(\mathrm{N})$ pulses of the voltage $V_{\mathrm{PA}}$, e.g., Figure $24 \mathrm{~b}$ shows a $V_{\mathrm{PA}}$ waveform when $d=1$, while Figure $24 \mathrm{c}$ shows the $d=0.5$ scenario, where some pulses are removed and denoted by " 0 ". Considering the root-mean-square (RMS) value of the $V_{\mathrm{PA}}$ fundamental component is:

$$
V_{\mathrm{PA}}=\frac{2 \sqrt{2}}{\pi} V D D \times d
$$

$V_{\mathrm{PA}}$ can be well-regulated with any specified $d$ within the range of $[0,1]$. The measured results showed that this scheme maintained a constant output power of $10 \mathrm{~W}$ and achieved a maximum efficiency from $93 \%$ to $73 \%$ when the coil distance varied from 0.1 to $0.4 \mathrm{~m}$ under a $100 \Omega$ load resistance. In addition, the gate control voltages $V_{\mathrm{G} 1 \sim 4}$ are controlled by a specially designed $\Delta \Sigma$-modulator to maintain the PA's stability.

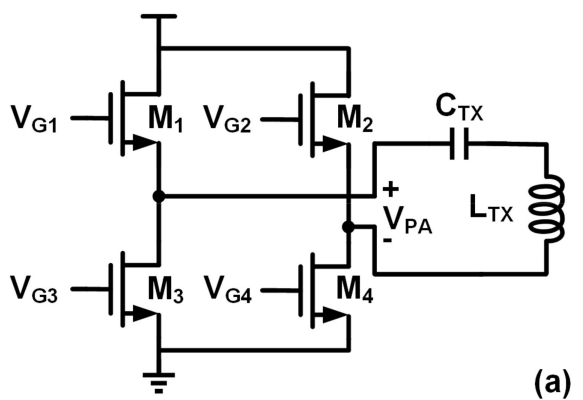

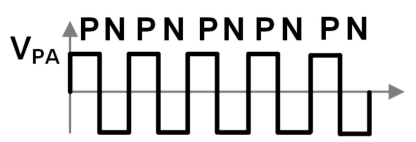

(b)

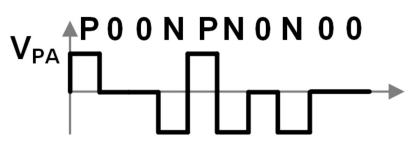

(c)

Figure 24. (a) The schematic of TX regulation using the PDM based on $\Delta \Sigma$-modulation, and the waveform of $V_{\mathrm{PA}}$ when (b) $d=1$ and (c) $d=0.5$ [38]. P, positive; $\mathrm{N}$, negative. 


\subsubsection{Phase-Shifted Modulation}

The authors in [36] presented a scheme that regulates the output voltage $V_{\mathrm{PA}}$ by varying the phase-shifted angle $\alpha$ of the gate control signals $V_{\mathrm{G} 1 \sim 4}$ in differential PA. As shown in Figure 25, the duty-cycle of each switching signal $V_{\mathrm{G} 1 \sim 4}$ is $50 \%$, but pulses of switch for $M_{4}$ and $M_{2}$ can lag $M_{1}$ and $M_{3}$ at a certain phase angle $\alpha$ ranging from $0^{\circ}$ to $180^{\circ}$. Hence, by increasing $\alpha$ between $V_{\mathrm{G} 1(2)}$ and $V_{\mathrm{G} 4(3)}$, the simultaneous conduction time $T_{14(23)}$ of the power transistors $M_{1(2)}$ and $M_{4(3)}$ will be reduced, and thus the $V_{\mathrm{PA}}$ will be decreased. The measured results demonstrated that the $V_{\mathrm{PA}}$ was regulated to $28 \mathrm{~V}$ (rms) when the supply voltage ranged from 35 to $75 \mathrm{~V}$, and the PA efficiency was around $94.5 \%$.

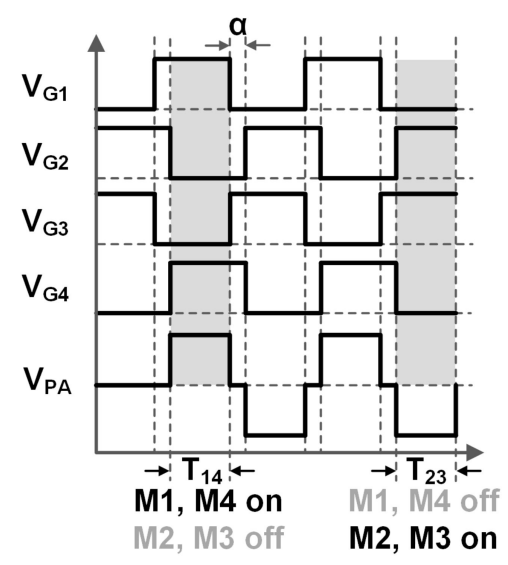

Figure 25. The waveforms of $V_{\mathrm{G} 1 \sim 4}, V_{\mathrm{PA}}$, and $I_{\mathrm{TX}}$ in phase-shifted modulation (PSM), where $\alpha$ is the phase-shifted angle [39].

However, the PA switching loss increases as the phase-shifted angle $\alpha$ approaches $180^{\circ}$, which results in a low efficiency under a light load condition. To address this, [40] demonstrated a TX regulation with harmonic-based phase-shifted modulation (HPSM). This technique applies the $n$th harmonic component of the switching frequency to regulate the transmitted power, as shown in Figure 26b, taking the 3rd HPSM as an example as follows.

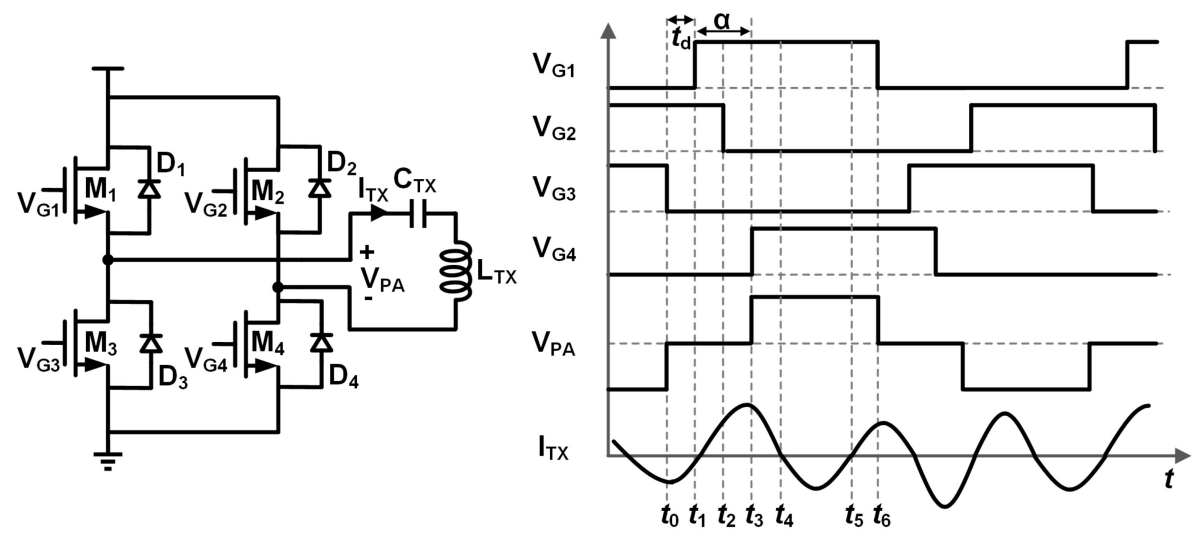

(a)

(b)

Figure 26. (a) The schematic of the differential PA and (b) the waveforms of $V_{\mathrm{G} 1-4}, V_{\mathrm{PA}}$, and $I_{\mathrm{TX}}$ in harmonic-based phase-shifted modulation (HPSM) with the 3rd harmonic [40].

A half-switching period is subdivided into six stages. Stage $1\left[t_{0}, t_{1}\right]: M_{2}$ is turned on, the power is oscillating freely through $M_{2}, L_{\mathrm{TX}}, C_{\mathrm{TX}}$, and $D_{1}$, and $V_{\mathrm{PA}}$ is equal to 0 . Stage $2\left[t_{1}, t_{2}\right]: M_{1}$ is turned on, the power is oscillating freely through $M_{1}, C_{\mathrm{TX}}, L_{\mathrm{TX}}$, and $D_{2}$, and $V_{\mathrm{PA}}$ is equal to 0 . Stage $3\left[t_{2}, t_{3}\right]: M_{2}$ is turned off, the power is oscillating freely through $M_{1}, C_{\mathrm{TX}}, L_{\mathrm{TX}}$, and $D_{2}$, and $V_{\mathrm{PA}}$ is still equal to 0 . 
Stage $4\left[t_{3}, t_{4}\right]: M_{4}$ is turned on, the power is transferred from the supply voltage to the ground through $M_{1}, C_{\mathrm{TX}}, L_{\mathrm{TX}}$, and $M_{4}$, and $V_{\mathrm{PA}}$ is equal to $V D D$. Stage $5\left[t_{4}, t_{5}\right]$ : the $I_{\mathrm{TX}}$ changes its direction, the power is circulated from the ground to the supply voltage through $D_{4}, L_{\mathrm{TX}}, C_{\mathrm{TX}}$, and $D_{1}$, and $V_{\mathrm{PA}}$ is equal to $V D D$. This stage finishes when $I_{\mathrm{TX}}$ reaches zero. Stage $6\left[t_{5}, t_{6}\right]$ : the $I_{\mathrm{TX}}$ changes its direction again, and the power is transferred from the supply voltage to the ground through $M_{1}, C_{\mathrm{TX}}, L_{\mathrm{TX}}$, and $M_{4}$. As a result, the current $I_{\mathrm{TX}}$ circulates three times during one switching period, which means lower switching loss compared with [39].

For higher-order harmonic control, there will be more current circulation per period and thus much lower switching loss, but smaller output power. The measurement results showed that HPSM with the 3rd harmonic component can improve PA efficiency by $10 \%$ compared to fundamental-based phase-shifted modulation.

\subsection{Vector Power Summing Control}

The aforementioned forms of TX regulation [32-40] are achieved only with a single TX, while it can also be implemented by summing the power from multiple TXs. The authors in [41,42] presented a vector power summing control technique to regulate the received power $P_{\text {total }}$ as demonstrated in Figure 27. In this scheme, two power vectors, implemented by separate TXs, are then summed at a single RX. The two TXs are driven by two switching clocks $\left(C L K_{1}\right.$ and $\left.C L K_{2}\right)$ with different phase $\theta$ under the same switching frequency $\left(f_{\mathrm{sw}}=1 / T_{\mathrm{sw}}\right)$. As a result, two transmitted powers, $P_{\mathrm{o}}$ and $P_{\mathrm{o}} \mathrm{e}^{\mathrm{j} \theta}$, are achieved in the two physically overlapped TX coils $L_{\mathrm{TX} 1}$ and $L_{\mathrm{TX} 2}$, respectively. Additionally, the transmitted power combined can be written as:

$$
P_{\text {total }}=P_{\mathrm{o}} \times(1+\cos \theta) .
$$

From Equation (13), the transmitted power can be regulated by controlling $\theta$ using a delay locked loop (DLL) and phase control circuit based on global feedback from the RX. In addition, wireless data communication can be simultaneously achieved using this vector summing technique [42].

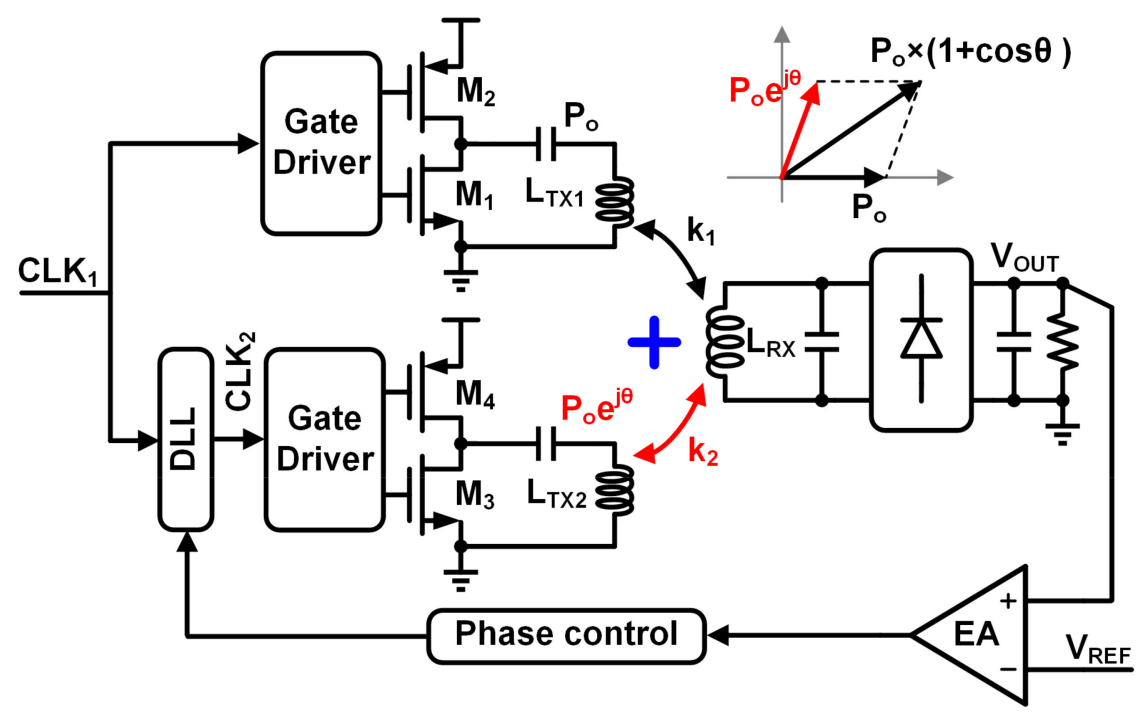

Figure 27. TX regulation based on the vector power summing control technique [41,42], where the output power is summed over two vector-transmitted powers. DLL, delay locked loop; EA, error amplifier.

Nevertheless, the vector summing technique will lead to a higher loss and system complexity due to the multiple TXs used. The measurement results showed that $V_{\text {OUT }}$ can be regulated from 3.3 to $16.3 \mathrm{~V}$ with $1 \mathrm{~W}$ output power and 50\% PTE. Also, boosting up to $20 \mathrm{~V}$ was achieved at a $0.6 \mathrm{~W}$ power 
transfer without a boost-type DC-DC converter or charge pump circuit. Additionally, a $35 \mu$ s response time was measured against a $1 \mathrm{~ms}$ load fluctuation interval.

Table 2 gives a comparison of the TX regulation topologies among state-of-the-art works, where all achieve reasonably good PTE. For the supply voltage control scheme, it is the most straightforward approach to TX regulation. However, the switching loss can be further reduced by maintaining a low PA switching frequency, while using its subharmonic component as the resonant frequency, as in the PA duty-cycle control scheme. For the other two schemes, the narrow ISM bandwidth limits the performance of the $f_{\text {res }}$ control scheme, while an additional TX requirement undermines the vector power summing control scheme.

Table 2. The comparison of TX regulation topologies in the state-of-the-art R-WPT systems.

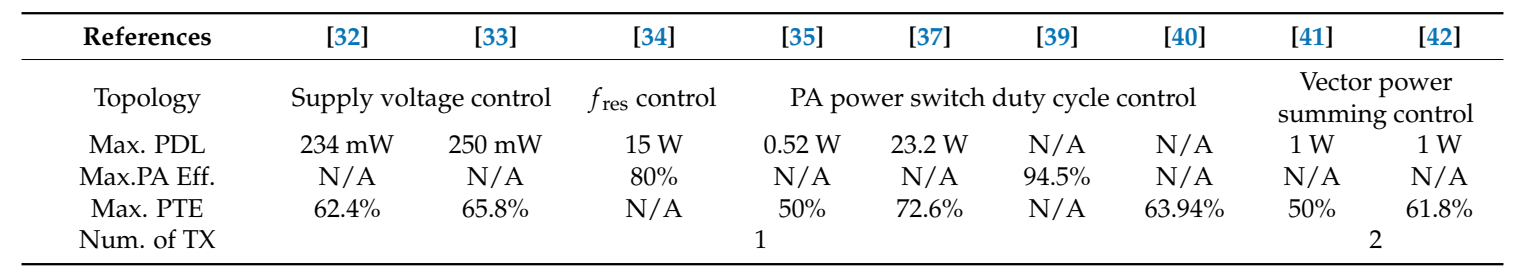

\section{Discussions and Development Trends}

For the resonant coupling WPT systems, as reviewed in this paper at the circuit level, output regulation can be achieved by post-stage regulation and one-stage regulation at the RX side as well as PA supply voltage control, resonant frequency control, duty-cycle control, and vector power summing control at the TX side.

For $\mathrm{RX}$ regulation, due to the poor efficiency of post-stage regulation, one-stage regulation, such as active diode conduction time control and $\mathrm{R}^{3}$ rectifier schemes, has been proposed for high PCE. However, the shortcoming of one-stage regulation is that the VCE is always smaller than 1.

Another one-stage regulation based on the switching-based current-mode (SCM) technique adopts the switching control method to utilize power transfer within two phases: store energy in the RX LC tank and then deliver the energy to the load. As a result, the voltage across the resonant $\mathrm{RX}$ tank can be boosted and this technique allows a $>1 \mathrm{VCE}$ at the RX side, which is thus suitable for a high-voltage application. Although the output regulation can be achieved by modulating the $R_{\text {eq, }}$ the PTE is still unoptimized.

There are two disadvantages for RX-only regulation schemes. For one thing, the RX output may fall outside of regulation considering a limited RX regulation range. For the other thing, the regulation may deviate the $R_{\text {refl }}$ from the one for an optimal PTE considering that the loading and coupling conditions vary. Hence, TX regulation that optimizes the PTE based on global feedback from the RX side is also necessary.

For TX regulation, the PA supply voltage control scheme, typically implemented with a DC-DC converter, is straightforward but has relatively low efficiency and high cost. Another TX regulation scheme is resonant frequency control, based on a binary-weighted capacitor array, but its low resolution and high cost limit its further application. PA switching duty-cycle control is an effective way to achieve a continuously adjustable output, but zero voltage/current switching is required for high efficiency. In addition, a vector power summing scheme can also fulfill output regulation, but the regulation accuracy and efficiency are not favorable.

Moreover, for all of the TX regulation schemes, a global control loop is required to feed the RX output information back to the TX side through a data uplink channel. As such, the load transient response speed depends not only on the bandwidth of the global feedback loop, but also the data rate of the uplink channel. The data uplink telemetry can be implemented either in wired $[17,35,41]$ or wireless ways. The wireless uplink schemes include Bluetooth, which has already be applied in A4WP [16,37], and backscattering based on load shift keying (LSK) $[24,32,33]$. 
To simplify the design of the binary-weighted capacitor array used in the resonant frequency control, a single phase switched fractional capacitor can be adopted for TX regulation by using the resonant frequency trimming technique as given in [45].

Another potential regulation topology is that the automatic maximum efficiency point tracking method [46] is implemented at the RX side and the regulation is achieved at the TX side. With this combined topology, the PTE can be optimized dynamically over a coupling and loading range with the help of TX regulation.

Last but not least, it is desirable to analyze the system stability and response speed because additional poles and zeros might be introduced with the regulation topologies.

\section{Conclusions}

In this work, the state-of-the-art regulation topologies in R-WPT systems for consumer electronics and bio-implants were comprehensively summarized and compared by mainly focusing on how to optimize the PTE, PCE, VCE, and PDL under loading or coupling variations. In addition, based on the advantages and drawbacks of these topologies, potential topologies are also proposed for researchers in this area. The regulation topologies design guidelines are not only suitable for R-WPT systems, but also for other near-field WPT systems.

Author Contributions: All authors conceived the work. The paper was written by Y.L. and revised by M.H. and B.L. The regulation topology classification and conclusion are suggested by Z.C. and X.Z.

Acknowledgments: This research is supported by the National Natural Science Foundation of China (61604044 and 61571196), the Fundamental Research Funds for the Central Universities (2017MS037), and the Science and Technology Project of Guangdong Province, China (2017B090908004, 2015B090901048).

Conflicts of Interest: The authors declare no conflict of interest.

\section{References}

1. Chiaraviglio, L.; D’Andreagiovanni, F.; Lancellotti, R.; Shojafar, M.; Melazzi, N.B.; Canali, C. An Approach to Balance Maintenance Costs and Electricity Consumption in Cloud Data Centers. IEEE Trans. Sustain. Comput. 2018, 1-15. [CrossRef]

2. Chiaraviglio, L.; Blefari-Melazzi, N.; Canali, C.; Cuomo, F.; Lancellotti, R.; Shojafar, M. A Measurement-Based Analysis of Temperature Variations Introduced by Power Management on Commodity Hardware. In Proceedings of the 2017 19th International Conference on Transparent Optical Networks (ICTON), Girona, Spain, 2-6 July 2017; pp. 1-4.

3. Mayordomo, I.; Drager, T.; Spies, P.; Bernhard, J.; Pflaum, A. An Overview of Technical Challenges and Advances of Inductive Wireless Power Transmission. Proc. IEEE 2013, 101, 1302-1311. [CrossRef]

4. Covic, G.A.; Boys, J.T. Inductive Power Transfer. Proc. IEEE 2013, 101, 1276-1289. [CrossRef]

5. Agarwal, K.; Jegadeesan, R.; Guo, Y.X.; Thakor, N.V. Wireless Power Transfer Strategies for Implantable Bioelectronics: Methodological Review. IEEE Rev. Biomed. Eng. 2017, 10, 136-161. [CrossRef] [PubMed]

6. Dai, J.; Ludois, D.C. A Survey of Wireless Power Transfer and a Critical Comparison of Inductive and Capacitive Coupling for Small Gap Applications. IEEE Trans. Power Electron. 2015, 30, 6017-6029. [CrossRef]

7. Jiang, C.; Chau, K.T.; Liu, C.; Lee, C.H.T. An Overview of Resonant Circuits for Wireless Power Transfer. Energies 2017, 10, 894. [CrossRef]

8. Zhou, D.D.; Greenbaum, E. Implantable Neural Prostheses 2: Techniques and Engineering Approaches; Springer: New York, NY, USA, 2010; ISBN 978-0-387-98119-2.

9. Ishida, K.; Yasufuku, T.; Miyamoto, S.; Nakai, H.; Takamiya, M.; Sakurai, T.; Takeuchi, K. 1.8 V Low-Transient-Energy Adaptive Program-Voltage Generator Based on Boost Converter for 3D-Integrated NAND Flash SSD. IEEE J. Solid-State Circuits 2011, 46, 1478-1487. [CrossRef]

10. Pagano, R.; Baker, M.; Radke, R.E. A 0.18- $\mu \mathrm{m}$ Monolithic Li-Ion Battery Charger for Wireless Devices Based on Partial Current Sensing and Adaptive Reference Voltage. IEEE J. Solid-State Circuits 2012, 47, 1355-1368. [CrossRef] 
11. Huang, M.; Lu, Y.; Seng-Pan, U.; Martins, R.P. 22.4 A Reconfigurable Bidirectional Wireless Power Transceiver with Maximum-Current Charging Mode and 58.6\% Battery-to-Battery Efficiency. In Proceedings of the IEEE International Solid-State Circuits Conference (ISSCC), Digest of Technical Papers, San Francisco, CA, USA, 5-9 February 2017; pp. 376-377.

12. Wu, C.Y.; Qian, X.H.; Cheng, M.S.; Liang, Y.A.; Chen, W.M. A 13.56 MHz 40 mW CMOS High-Efficiency Inductive Link Power Supply Utilizing On-Chip Delay-Compensated Voltage Doubler Rectifier and Multiple LDOs for Implantable Medical Devices. IEEE J. Solid-State Circuits 2014, 49, 2397-2407. [CrossRef]

13. Lee, H. An Auto-Reconfigurable AC-DC Regulator for Wirelessly Powered Biomedical Implants With $28 \%$ Link Efficiency Enhancement. IEEE Trans. Very Large Scale Integr. VLSI Syst. 2016, 24, 1598-1602. [CrossRef]

14. Kiani, M.; Lee, B.; Yeon, P.; Ghovanloo, M. 12.7 A Power-Management ASIC with Q-modulation Capability for Efficient Inductive Power Transmission. In Proceedings of the IEEE International Solid-State Circuits Conference (ISSCC), Digest of Technical Papers, San Francisco, CA, USA, 22-26 February 2015; pp. 226-228.

15. Lee, H.M.; Ghovanloo, M. An Integrated Power-Efficient Active Rectifier with Offset-Controlled High-Speed Comparators for Inductively Powered Applications. IEEE Trans. Circuits Syst. Regul. Pap. 2011, 58, 1749-1760. [CrossRef] [PubMed]

16. Moh, K.G.; Neri, F.; Moon, S.; Yeon, P.; Yu, J.; Cheon, Y.; Roh, Y. s; Ko, M.; Park, B.H. 12.9 A Fully Integrated 6W Wireless Power Receiver Operating at $6.78 \mathrm{MHz}$ with Magnetic Resonance Coupling. In Proceedings of the IEEE International Solid-State Circuits Conference (ISSCC), Digest of Technical Papers, San Francisco, CA, USA, 22-26 February 2015; pp. 230-232.

17. Choi, J.-H.; Yeo, S.-K.; Park, S.; Lee, J.-S.; Cho, G.-H. Resonant Regulating Rectifiers (3R) Operating for 6.78 MHz Resonant Wireless Power Transfer (RWPT). IEEE J. Solid-State Circuits 2013, 48, 2989-3001. [CrossRef]

18. Park, Y.J.; Jang, B.; Park, S.M.; Ryu, H.C.; Oh, S.J.; Kim, S.Y.; Pu, Y.; Yoo, S.S.; Hwang, K.C.; Yang, Y.; et al. A Triple-Mode Wireless Power-Receiving Unit With 85.5\% System Efficiency for A4WP, WPC, and PMA Applications. IEEE Trans. Power Electron. 2018, 33, 3141-3156. [CrossRef]

19. Moon, Y.J.; Roh, Y.S.; Yoo, C.; Kim, D.Z. A 3.0-W Wireless Power Receiver Circuit with 75-\% Overall Efficiency. In Proceedings of the IEEE Asian Solid-State Circuits Conference (A-SSCC), Kobe, Japan, 12-14 November 2012; pp. 97-100.

20. Park, H.G.; Jang, J.H.; Kim, H.J.; Park, Y.J.; Oh, S.; Pu, Y.; Hwang, K.C.; Yang, Y.; Lee, K.Y. A Design of a Wireless Power Receiving Unit with a High-Efficiency 6.78-MHz Active Rectifier Using Shared DLLs for Magnetic-Resonant A4WP Applications. IEEE Trans. Power Electron. 2016, 31, 4484-4498. [CrossRef]

21. Kim, C.; Ha, S.; Park, J.; Akinin, A.; Mercier, P.P.; Cauwenberghs, G. A 144-MHz Fully Integrated Resonant Regulating Rectifier with Hybrid Pulse Modulation for Mm-Sized Implants. IEEE J. Solid-State Circuits 2017, 52, 3043-3055. [CrossRef]

22. Lee, H.M.; Park, H.; Ghovanloo, M. A Power-Efficient Wireless System with Adaptive Supply Control for Deep Brain Stimulation. IEEE J. Solid-State Circuits 2013, 48, 2203-2216. [CrossRef] [PubMed]

23. Ozalevli, E.; Femia, N.; Capua, G.D.; Subramonian, R.; Du, D.; Sankman, J.; Markhi, M.E. A Cost-Effective Adaptive Rectifier for Low Power Loosely Coupled Wireless Power Transfer Systems. IEEE Trans. Circuits Syst. Regul. Pap. 2017, 1-12. [CrossRef]

24. Li, X.; Tsui, C.-Y.; Ki, W.-H. A 13.56 MHz Wireless Power Transfer System with Reconfigurable Resonant Regulating Rectifier and Wireless Power Control for Implantable Medical Devices. IEEE J. Solid-State Circuits 2015, 50, 978-989. [CrossRef]

25. Cheng, L.; Ki, W.H.; Tsui, C.Y. A 6.78-MHz Single-Stage Wireless Power Receiver Using a 3-Mode Reconfigurable Resonant Regulating Rectifier. IEEE J. Solid-State Circuits 2017, 52, 1412-1423. [CrossRef]

26. Lee, H.M.; Ghovanloo, M. An Adaptive Reconfigurable Active Voltage Doubler/Rectifier for Extended-Range Inductive Power Transmission. In Proceedings of the IEEE International Solid-State Circuits Conference (ISSCC), Digest of Technical Papers, San Francisco, CA, USA, 19-23 February 2012; pp. $286-288$.

27. Lu, Y.; Li, X.; Ki, W.H.; Tsui, C.Y.; Yue, C.P. A $13.56 \mathrm{MHz}$ Fully Integrated 1X/2X Active Rectifier with Compensated Bias Current for Inductively Powered Devices. In Proceedings of the IEEE International Solid-State Circuits Conference (ISSCC), Digest of Technical Papers, San Francisco, CA, USA, 17-21 February 2013; pp. 66-67.

28. Kiani, M.; Lee, B.; Yeon, P.; Ghovanloo, M. A Q-Modulation Technique for Efficient Inductive Power Transmission. IEEE J. Solid-State Circuits 2015, 50, 2839-2848. [CrossRef] [PubMed] 
29. Ahn, D.; Kim, S.; Moon, J.; Cho, I.-K. Wireless Power Transfer with Automatic Feedback Control of Load Resistance Transformation. IEEE Trans. Power Electron. 2016, 31, 7876-7886. [CrossRef]

30. Lee, B.; Yeon, P.; Ghovanloo, M. A Multicycle Q-Modulation for Dynamic Optimization of Inductive Links. IEEE Trans. Ind. Electron. 2016, 63, 5091-5100. [CrossRef] [PubMed]

31. Gougheri, H.S.; Kiani, M. Current-Based Resonant Power Delivery with Multi-Cycle Switching for Extended-Range Inductive Power Transmission. IEEE Trans. Circuits Syst. Regul. Pap. 2016, 63, 1543-1552. [CrossRef]

32. Li, X.; Tsui, C.Y.; Ki, W.H. 12.8 Wireless Power Transfer System Using Primary Equalizer for Coupling- and Load-Range Extension in Bio-implant Applications. In Proceedings of the IEEE International Solid-State Circuits Conference (ISSCC), Digest of Technical Papers, San Francisco, CA, USA, 22-26 February 2015; pp. 228-230.

33. Wang, G.; Liu, W.; Sivaprakasam, M.; Kendir, G.A. Design and Analysis of an Adaptive Transcutaneous Power Telemetry for Biomedical Implants. IEEE Trans. Circuits Syst. Regul. Pap. 2005, 52, 2109-2117. [CrossRef]

34. Si, P.; Hu, A.P.; Malpas, S.; Budgett, D. A Frequency Control Method for Regulating Wireless Power to Implantable Devices. IEEE Trans. Biomed. Circuits Syst. 2008, 2, 22-29. [CrossRef] [PubMed]

35. Shinoda, R.; Tomita, K.; Hasegawa, Y.; Ishikuro, H. Voltage-Boosting Wireless Power Delivery System with Fast Load Tracker by $\Delta \Sigma$-modulated Sub-Harmonic Resonant Switching. In Proceedings of the IEEE International Solid-State Circuits Conference (ISSCC), Digest of Technical Papers, San Francisco, CA, USA, 19-23 February 2012; pp. 288-290.

36. Li, X.; Li, Y.P.; Tsui, C.Y.; Ki, W.H. Wireless Power Transfer System with $\Delta \Sigma$-Modulated Transmission Power and Fast Load Response for Implantable Medical Devices. IEEE Trans. Circuits Syst. II Express Briefs 2017, 64, 279-283. [CrossRef]

37. Zhong, W.; Hui, S.Y.R. Maximum Energy Efficiency Operation of Series-Series Resonant Wireless Power Transfer Systems Using On-Off Keying Modulation. IEEE Trans. Power Electron. 2018, 33, 3595-3603. [CrossRef]

38. Li, H.; Wang, K.; Fang, J.; Tang, Y. Pulse Density Modulated ZVS Full-Bridge Converters for Wireless Power Transfer Systems. IEEE Trans. Power Electron. 2018. [CrossRef]

39. Ye, Z.; Jain, P.K.; Sen, P.C. A Full-Bridge Resonant Inverter with Modified Phase-Shift Modulation for High-Frequency AC Power Distribution Systems. IEEE Trans. Ind. Electron. 2007, 54, 2831-2845. [CrossRef]

40. Cai, H.; Shi, L.; Li, Y. Harmonic-Based Phase-Shifted Control of Inductively Coupled Power Transfer. IEEE Trans. Power Electron. 2014, 29, 594-602. [CrossRef]

41. Tomita, K.; Shinoda, R.; Kuroda, T.; Ishikuro, H. 1W 3.3V-to-16.3V Boosting Wireless Power Transfer Circuits with Vector Summing Power Controller. IEEE J. Solid-State Circuits 2012, 47, 2576-2585. [CrossRef]

42. Kosuge, A.; Hashiba, J.; Kawajiri, T.; Hasegawa, S.; Shidei, T.; Ishikuro, H.; Kuroda, T.; Takeuchi, K. An Inductively Powered Wireless Solid-State Drive System with Merged Error Correction of High-Speed Wireless Data Links and NAND Flash Memories. IEEE J. Solid-State Circuits 2016, 51, 1041-1050. [CrossRef]

43. Van Schuylenbergh, K.; Puers, R. Inductive Powering: Basic Theory and Application to Biomedical Systems. In Analog Circuits and Signal Processing; Springer: Dordrecht, The Netherlands, 2009; ISBN 978-90-481-2411-4.

44. Finkenzeller, K. Fundamentals and Applications in Contactless Smart Cards, Radio Frequency Identification and Near-Field Communication, 3rd ed.; Wiley: Chichester, UK; Hoboken, NJ, USA, 2010; ISBN 978-0-470-69506-7.

45. Kennedy, H.; Bodnar, R.; Lee, T.; Redman-White, W. A self-tuning resonant inductive link transmit driver using quadrature-symmetric phase-switched fractional capacitance. In Proceedings of the IEEE International Solid-State Circuits Conference (ISSCC), Digest of Technical Papers, San Francisco, CA, USA, 5-9 February 2017; pp. 370-371.

46. Mao, F.; Lu, Y.; Seng-Pan, U.; Martins, R.P. A Reconfigurable Cross-Connected Wireless-Power Transceiver for Bidirectional Device-to-Device Charging with 78.1\% Total Efficiency. In Proceedings of the IEEE International Solid-State Circuits Conference (ISSCC), Digest of Technical Papers, San Francisco, CA, USA, 11-15 February 2018; pp. 140-142.

(C) 2018 by the authors. Licensee MDPI, Basel, Switzerland. This article is an open access article distributed under the terms and conditions of the Creative Commons Attribution (CC BY) license (http:/ / creativecommons.org/licenses/by/4.0/). 